\title{
Review
}

Fei Xing, Changchun Zhou*, Didi Hui, Colin Du, Lina Wu, Linnan Wang, Wenzhao Wang, Xiaobing Pu, Linxia Gu, Lei Liu, Zhou Xiang, and Xingdong Zhang

\section{Hyaluronic acid as a bioactive component for bone tissue regeneration: Fabrication, modification, properties, and biological functions}

https://doi.org/10.1515/ntrev-2020-0084

received September 2, 2020; accepted September 23, 2020
Keywords: bioactive component, hyaluronic acid, bone regeneration, 3D printing, tissue engineering

\section{Introduction}

Hyaluronic acid (HA), also known as hyaluronan or hyaluronate, exists in all body fluids and the extracellular matrix (ECM) of many tissues [1,2]. It is responsible for regulating tissue hydration and adjusting the viscoelasticity of some body fluids, such as synovial fluid in the joint cavity [3]. It also regulates fundamental cellular processes, including cell adhesion, proliferation, and differentiation, by binding with surface receptors of target cells $[4,5]$. In addition, HA participates in many biological responses, including angiogenesis, inflammation, wound healing, and bone regeneration [6,7]. HA-based medical productions have been in clinical use in drug delivery, cosmetics, and tissue regeneration for over 40 years because of their outstanding biodegradability and biocompatibility properties $[8,9]$.

At present, the repair of large bone defects due to infection, trauma, surgical resection of tumor, and malformation still remains a major challenge in modern medicine $[8,9]$. The gold standard for the treatment of large bone defects is bone transplantation, including autografting and allografting [10-12]. However, many problems arise with this treatment approach, such as limited sources, immunological rejection, infection, donor-site morbidity, and graft resorption [13,14]. Bone substitutes fabricated by the tissue-engineered methods are rapidly becoming promising alternatives for the treatment of bone defects $[15,16]$. In recent years, given the increasing demand for the repair and replacement of bone tissue, various tissue-engineered bone scaffolds loaded with or without cells have been fabricated for bone regeneration. Owing to its favorable biochemical properties, HA, as well as other common natural polymers such as alginate, 
collagen, gelatin, silk fibroin, fibrin, and chitosan, has already been utilized to construct various tissue-engineered bone scaffolds in the field of bone repair with a rich research history [17]. As a natural component of ECM, HA can not only provide osteogenesis-related cells with a similar extracellular environment but also initiate many cellular signaling pathways in bone regeneration $[18,19]$. Additionally, the biological function and physicochemical properties of HA can be changed by chemical modification [20]. HA and its derivatives are also commonly used to mix with other gels, resulting in a hybrid hydrogel with combined characteristics. By combining with various tissue-engineered processing techniques such as freeze drying, electrospinning, and 3D printing, bone substitutes based on HA and its derivatives offer the versatility to be modified into any shapes or sizes including conversion to porous scaffolds, nanofibers, films, nanoparticles, and microspheres, for bone tissue regeneration [21,22]. Even though $\mathrm{HA}$ itself has a limited osteogenic effect in the process of bone repair, $\mathrm{HA}$ and its derivatives can be used as vehicles for osteogenesis-related cells and factors or utilized in combination with other biomaterials such as ceramics and titanium implants for bone regeneration. Therefore, we conduct this review to discuss the current status and role of HA applied in bone tissue engineering. Furthermore, the modification of HA and processing techniques of HA-based biomaterials are also discussed.

\section{Properties and biological functions of $\mathrm{HA}$}

\subsection{Structure and properties of HA}

HA is an anionic, nonsulfated, and simplest glycosaminoglycan (GAG) and composed of repeating units of D-glucuronic acid and $\mathrm{N}$-acetylglucosamine combined by $\beta-1,4$ and $\beta-1,3$-glycosidic bonds [23]. The number of repeating units in HA can reach about 10,000 or more, resulting in a molecular weight of 4 million daltons $(\mathrm{Da})$. HA can bind and retain a large amount of water molecules because it is rich in negatively charged hydroxyl groups. The chemical structure of HA is shown in Figure 1. Different from other GAGs, HA is synthesized by hyaluronan synthase enzymes (HAS1, HAS2, and HAS3) in the cell membrane, instead of the Golgi apparatus, and does not attach to a core protein [24]. HA has a high molecular weight, ranging from $10^{5} \mathrm{Da}$ in serum to $10^{7} \mathrm{Da}$ in vitreous,

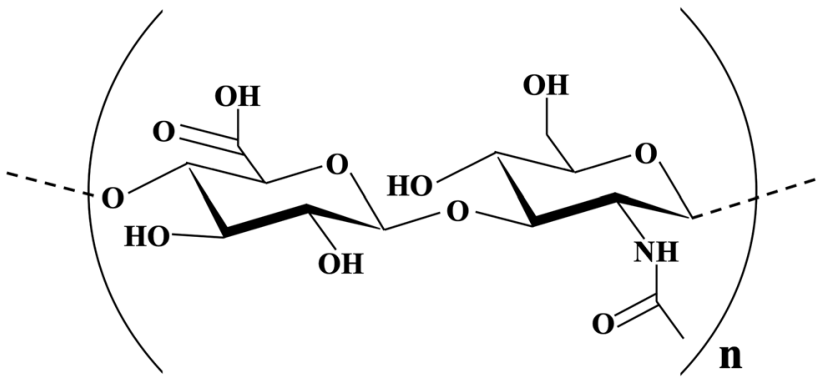

Figure 1: The chemical structure of HA. Repeating sugar molecules form the backbone of the HA chain.

and is rapidly degraded in the tissue by hyaluronidase; specifically, $N$-acetylhexosaminidase and D-glucuronidase catalyze the cleavage of glycosidic bonds to remove nonreducing terminal sugars, degrading high molecular weight HA into smaller fragments [23].

As a long-chain biopolymer in solution, HA can appear as a reinforced random coil structure with a large hydration volume, forming a stiff, viscous, gelatin-like substance by each molecule interacting with its neighbors [25]. HA is polydisperse in solution and can restrict the access of other macromolecules into its domain, which demonstrates the excluded volume effects of HA. Many current studies have demonstrated that hydrogen bonds between adjacent saccharides restrict the rotation of the glycosidic bond and contribute to the stiffening of the HA chain $[26,27]$. Therefore, HA forms a dynamic network, which can allow molecules with low molecular weight to penetrate freely and restrict the movements of other macromolecules with high molecular weight [25]. The solutions of HA under physiological conditions are highly viscoelastic, and the individual chains of HA remain mobile. Hence, HA can form higher order structures by interactions or combination with other proteins.

\subsection{Biological functions of $\mathrm{HA}$}

Many studies demonstrated that HA plays an important role in cell behavior regulation, including cell proliferation, survival, motility, migration, and differentiation $[28,29]$. As a transmembrane receptor for HA, CD44 is highly expressed by many cells to initiate various intracellular signaling cascades. By activating CD44-mediated pathways, HA can regulate the behaviors of osteogenesisrelated cells, such as cell adhesion and migration [30]. As another receptor of HA, receptor for hyaluronic acidmediated motility (RHAMM) has multiple isoforms and can alter migratory cell behavior [31]. Additionally, the 
different molecular weight and concentration of HA have different effects on cell proliferation and differentiation $[29,32]$. HA with low molecular weight $\left(<10^{3} \mathrm{kDa}\right)$ is mostly reported to increase cell proliferation and differentiation [33]. However, the effects of HA with high molecular weight on cell proliferation and differentiation remain controversial $[34,35]$.

HA also has bacteriostatic effect and anti-adhesive ability. Toll-like receptors (TLRs), single-pass membranespanning receptors, play a vital role in activating immune cell responses. HA fragments with low molecular weight can bind to TLR-4 and act as initiators for defending against bacterial infection [36]. Additionally, as a cell surface receptor of HA, ICAM-1 (intracellular adhesion molecule-1) is a member of the immunoglobulin superfamily that is expressed by lymphocytes and macrophages [37]. Currently, HA has been confirmed of its antimicrobial effects on Aggregatibacter actinomycetemcomitans, b-hemolytic Streptococcus, Prevotella oris, Enterococcus, Staphylococcus aureus, and Pseudomonas aeruginosa. Additionally, Drago et al. demonstrated that HA could interfere with bacterial adhesion and bacterial biofilm formation [38].

A previous study also found that HA could induce peripheral blood monocyte to express anti-inflammatory growth factors [39]. The effects of HA on anti-inflammatory functions depend on its molecular weight. HA with high molecular weight has anti-inflammatory effect, whereas low molecular weight degradation products of HA can induce various pro-inflammatory responses [40]. Additionally, HA fragments can induce macrophages to express nitric oxide, monocyte chemotactic protein-1, and macrophage inflammatory proteins [39]. Previous studies also found that HA with low molecular weight could induce the expression of inducible nitric oxide synthase (iNOS) in chondrocytes [41].

\subsection{Effects of HA on bone regeneration}

Bone tissue is a dense connective tissue consisting of $30 \%$ organic material and approximately $70 \%$ inorganic material. The process of bone repair includes hematoma formation, the inflammatory phase, granulation tissue formation, callus formation, and the remodeling phase [42]. Furthermore, osteogenesis-related cells, inflammatory cells, endothelial cells (ECs), and various growth factors are involved in the process of bone formation and bone remodeling. HA promotes bone regeneration by regulating cell activity and the release of biological factors. Additionally, the beneficial osteogenic effects of
HA have been demonstrated by many studies. HA can bind with CD44 and be incorporated into the cytoplasm of osteoprogenitor cells to regulate the osteoprogenitor cell migration [18]. Hempel et al. found that HA could promote osteogenic differentiation of mesenchymal stem cells (MSCs) even without the existence of dexamethasone [35]. In addition, HA with different molecular weights might have different biological functions during the process of bone regeneration. Zhao et al. found that HA with low molecular weight could enhance the proliferation of bone marrow-derived mesenchymal stem cells (BMSCs), whereas HA with high molecular weight could enhance mRNA expressions of osteogenic gene markers, such as ALP, RUNX2, and OCN [32]. Additionally, due to its good physicochemical properties, HA also can be used as a vehicle for osteogenesis-related cells and factors for bone repair.

Bone is a complex heterogeneous and vascularized tissue with vascular networks, which is connected to the blood system by transverse channels [43]. Metabolic needs and oxygen are not met when the length of bone substitutes exceeds $200 \mu \mathrm{m}$, resulting in core ischemia of bone substitutes and poor integration with host tissue [44]. Moreover, insufficient vascularization of bone substitutes often results in poor bone regeneration. Additionally, angiogenesis plays a vital role in the process of bone formation [45]. The fragments of HA can enhance angiogenesis via RHAMM-mediated signaling pathways in epithelial cells [46]. Additionally, HA is also responsible for promoting the proliferation and migration of ECs in the process of vessel formation [47]. Wang et al. found that HA could enhance the capillary density and blood supply during the process of tissue repair in vivo [48]. Ciccone et al. found that when ECs are exposed to HA, the expression of vascular endothelial growth factor (VEGF) is enhanced [49]. In another study, Matsumoto et al. utilized freeze drying technique to fabricate different sponges using HA with diverse molecular weights [50]. The in vivo results demonstrated that the combination of HA with low molecular weight on the sponges had a positive effect on the angiogenesis process.

\section{Chemical modifications and crosslinking of HA}

HA is an attractive component of artificial biomaterial fabrication in the field of tissue engineering due to its biodegradability, biocompatibility, non-immunogenicity, and non-thrombogenicity. However, native HA cannot be 
a useful biomaterial for tissue regeneration owing to its poor mechanical properties and susceptibility to degradation in vivo. High molecular weight HA solution with high concentration is viscoelastic, and native HA molecules interact with each other by H-bonding, Van der Waals interactions, and hydrophobic forces in dilute physiological solution. However, the HA network is heterogeneous, unstable, and reversible, exhibiting weak mechanical property [23]. In fact, native HA cannot maintain its structural integrity in vivo and in vitro. Environmental factors in vivo, such as $\mathrm{pH}$ and temperature, can also change its properties [51]. HA can dissolve in aqueous solution and be degraded by hyaluronidase quickly in physiological milieu [52]. The tissue half-lives of HA are short, ranging from minutes in the blood to hours or days in skin and joints [53]. Moreover, HA is metabolized to oligosaccharides $(<10 \mathrm{kDa})$ and low (4-100 kDa) or high molecular weight fragments $(>100 \mathrm{kDa})$, resulting in decreased viscoelasticity of the HA solution.

In the past several decades, for better application of HA in bone regeneration, many researchers have developed various innovative technologies of hydrophobic modification to improve the properties of HA [23]. Hydrophobic modification of HA can be achieved by chemical modification of multiple functional groups [54]. Additionally, in situ photopolymerization can convert some kinds of liquid HA biomaterials, which contain more than one reactive group on the HA macromer, into solid constructs by exposure to light [20]. This section reviews the different methods of modified HA reported in the literature in the late years.

\subsection{Chemical modifications of HA}

\subsubsection{Hydrazide modification}

HA can be modified with adipic dihydrazide, resulting in hydrazide-modified HA. The chemical structure of hydrazide-modified HA is shown in Figure 2a. Chen et al. utilized hydrazide-modified HA to fabricate a biomimetic hydrogel system, whose physicochemical properties can be adjusted by varying the weight ratio of polysaccharides [55]. In another study, Wang et al. fabricated an injectable hydrogel by hydrazide-modified HA, which has an outstanding mechanical property to protect encapsulated human MSCs in hydrogel during the procedure of injection and maintain their osteogenic differentiation capability [56] (Figure 2b). In another study, Ossipov et al. successfully synthesized hydrazide-functionalized HA by initial mild cleavage of a disulfide bond and elimination of the generated 2-thioethoxycarbonyl moiety [57]. Additionally, hydrazide-functionalized HA can be used for continuous controlled release of osteogenesis-related factors during the process of bone repair [58]. Hydrazide-functionalized (a)

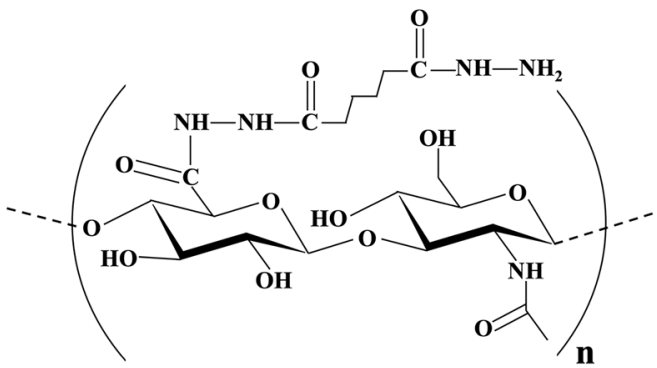

(b)

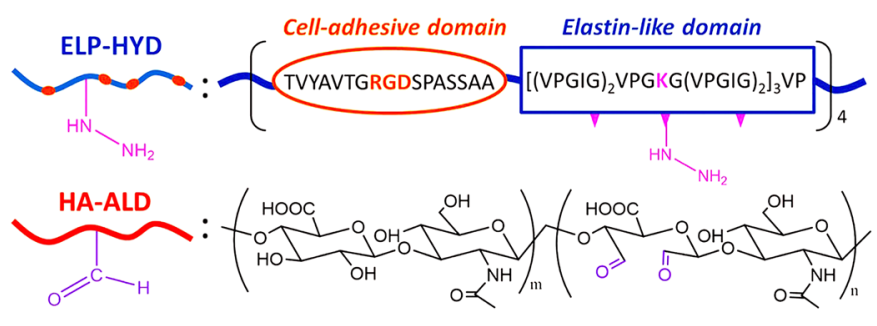

(c)

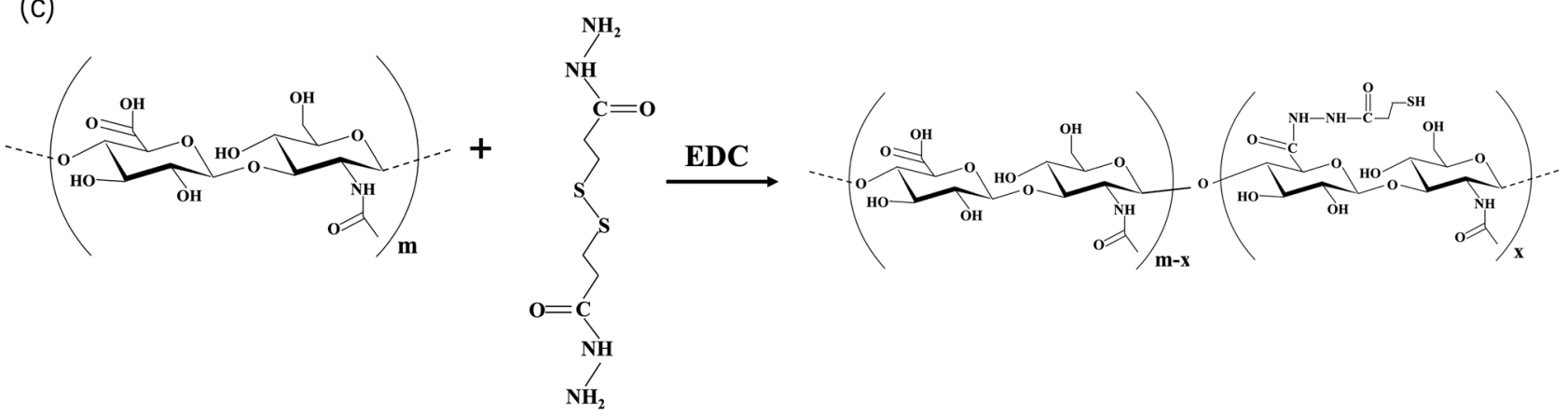

Figure 2: (a) The chemical structure of hydrazide-functionalized HA. (b) The structure of hydrazine-modified elastin-like protein and aldehyde-modified HA [56]. (c) Synthetic route of thiol-modified HA. 
HA not only has the basic biological functions of HA but also can further bind with various polypeptides to enhance osteogenic differentiation [58]. Additionally, compared with HA, hydrazide-functionalized HA has better physicochemical properties and drug resistance.

\subsubsection{Thiol modification}

Thiol groups can be introduced to HA by using dihydrazide reagents containing a disulfide bond in the presence of 1-ethyl-3-(3-dimethylaminopropyl) carbodiimide hydrochloride [59] (Figure 2c). Hosack et al. utilized thiolmodified HA, gelatin, and heparin to fabricate a hydrogel system preloaded with platelet-derived growth factor (PDGF), keratinocyte growth factor, angiopoietin-1, and VEGF [60]. After being implanted into the Balb/c mouse ear pinna, the results in vivo found the new microvessel development and maturation at 7 days postsurgery [60]. Additionally, thiol-modified HA can crosslink spontaneously to form a hydrogel, which could be used to fabricate tissueengineered bone scaffolds with porous structure and spongelike properties after the process of freeze drying [61]. Moreover, Kazemirad et al. utilized thiol-modified HA and gelatin to construct new synthetic ECM hydrogels, whose mechanical properties could be altered by changing concentrations of the constituents [62]. Multifunctional electrophiles, such as poly(ethylene glycol) diacrylate, can be crosslinked with thiol-modified HA to improve the biocompatibility of hydrogel [63]. Additionally, thiol-modified HA can combine with ECM proteins and improve cell survival and differentiation [64].

\subsubsection{Tyramine modification}

Tyramine-modified HA was synthesized by amide bond formation between amine groups of tyramine and carboxyl groups of HA [65]. The synthetic route of tyramine-modified HA is shown in Figure 3a. Kim et al. synthesized tyramine-modified HA by $N$-hydroxysulfosuccinimide and 1 ethyl-3-[3-(dimethylamino)propyl] carbodiimide and utilized radical crosslinking reaction with $\mathrm{H}_{2} \mathrm{O}_{2}$ and horseradish peroxidase to fabricate hydrogel [66] (Figure 3b). Many osteogenesis-related cells and factors can be incorporated into tyramine-modified HA hydrogel for bone regeneration. Zhang et al. constructed an injectable hydrogel system loaded with BMSCs and bone morphogenetic protein- 2 for bone repair and regeneration by enzymatic in situ crosslinking of HA-tyramine and chondroitin sulfate-tyramine in the presence of hydrogen peroxide and horseradish peroxidase [67] (Figure 3c). The results demonstrated that the injectable hydrogel system promoted bone regeneration [67]. The platelet lysate obtained from the peripheral blood can enhance cell attachment, viability, and chondrogenic differentiation. In another study, Jooybar et al. utilized the same method to incorporate platelet lysate into a cell-laden injectable HA-tyramine hydrogel [68]. The results in vivo found that the cells attached and spread out in HA-tyramine hydrogel. Additionally, tyramine-modified HA itself can also regulate MSCs' early attachment and behavior by activating related signal pathways [66]. However, there are still few studies focusing on the direct application of tyramine-modified HA to bone repair, and its specific bone repair mechanisms still need further research.

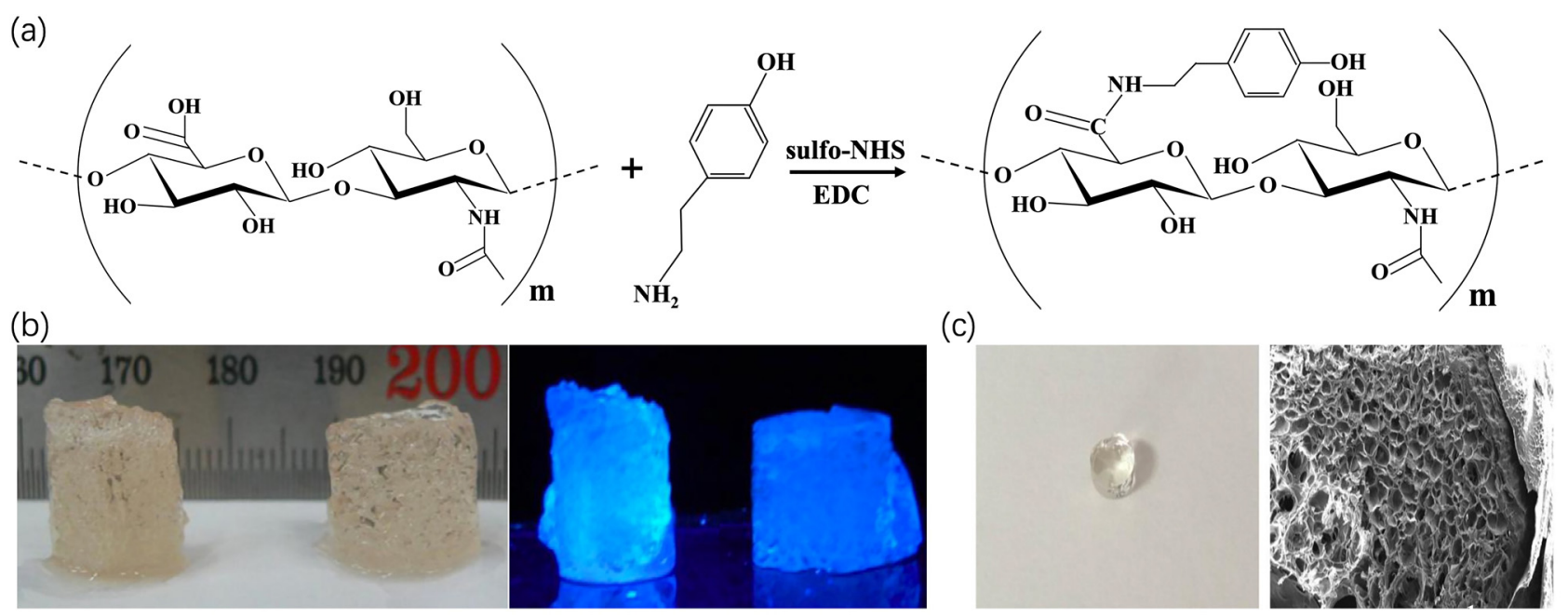

Figure 3: (a) The synthetic route of tyramine-modified HA. (b) The tyramine-modified HA hydrogels with and without exposure to UV light [66]. (c) The hydrogels composed of HA-tyramine and chondroitin sulfate-tyramine [67]. 
After chemical modification, the chemical and mechanical properties of HA can be enhanced, while its native biological functions can be maintained. Additionally, chemical modification of $\mathrm{HA}$ can also alter its resistance against enzymatic degradation and delay aqueous dissolution. Currently, hydrazide modification has been widely used in the chemical modification of HA because of its high cytocompatibility, efficiency, simplicity, reversibility, and mild reaction conditions [69]. In addition, hydrazide modification does not lead to cleavage of the HA chain. The thiol modification of HA has a high yield, efficiency, and a fast reaction rate. Compared with other kinds of chemical modification, thiol modification has a higher specificity [70]. Tyramine modification has advantages of mild reaction condition and a fast gelation rate. However, there are few studies focusing on the difference between various chemical modifications. Thus, the difference between various chemical modifications still needs further research.

\subsection{Crosslinking of HA}

Currently, crosslinking by means of radical polymerization has been widely applied in the in situ formation of HA-based hydrogels in the field of bone regeneration. Radical polymerization can react in the presence of aqueous solutions and control the chemical reactions [71]. Radical polymerization involves the placement of liquid biomaterials in the region of interest and its subsequent conversion into a solid implant, in the presence of crosslinking agents and some initiation source [72]. The initiators, such as photoinitiator in the presence of light, redox pair, and temperature, can induce the formation of radicals, which can react with reactive groups on the HA to form kinetic chains. Among these initiators, photoinitiated polymerizations are the most commonly used methods owing to their temporal and spatial control by light-triggered polymerization on the hydrogel formation. The biological properties of HA-based hydrogels can be adjusted by varying the molecular weight of HA, the concentration of the macromer, and the degree of methacrylation. In addition, photoinitiated polymerizations can also be applied in the direct cellular or protein encapsulation via altering radical concentrations or light intensities at a mild initiation condition. Currently, methacrylates degraded from methacrylic anhydride and glycidyl methacrylate in aqueous media are the most commonly used groups for HA-based hydrogel preparation.

\subsubsection{Crosslinking with glycidyl methacrylate}

Glycidyl methacrylate can react with HA to form a methacrylated HA (MeHA). Postoperative adhesions often remain permanent and complicate otherwise successful surgeries by tethering tissues together that are normally separated. An ideal adhesion barrier should prevent unwanted adhesions. Li et al. synthesized glycidyl methacrylate-hyaluronic acid (GMHA) conjugates by interacting glycidyl methacrylate with $\mathrm{HA}$ at room temperature, and then graft GMHA onto the surface of biomedical elastomer [73]. The result demonstrated that the GMHA could resist platelet adhesion and improve the proliferation of murine osteoblastic cell line MC-3T3-E1. In another study, Mayes et al. utilized alginate and GMHA to fabricate a robust hydrogel film, which showed a good ability of anti-adhesion in a rat peritoneal abrasion model for adhesion formation [74] (Figure 4a). Ma et al. utilized GMHA and poly( $y$-glutamic acid) to fabricate an injectable hydrogel, which showed excellent anti-compression ability and outstanding shape recovery capability [75]. Moreover, researchers can modulate the mechanical properties of hydrogel by varying the ratio of poly $(\gamma$-glutamic acid) and GMHA. Many researchers incorporated cell or growth factors into GMHA for tissue engineering. Zhang et al. incorporated BMSCs and BMP-2 into GMHA to form hydrogels by photoinitiated polymerizations for bone regeneration [76] (Figure 4b). In another study, Khoshakhlagh et al. employed GMHA and puramatrix to form a structurally adjustable interpenetrating network system [77]. Hsieh et al. constructed a tissue-engineered scaffold for bone regeneration by using methoxy poly (ethylene glycol)-block-poly(e-caprolactone), hydroxyapatite, and GMHA [78].

\subsubsection{Crosslinking with methacrylic anhydride}

The reaction of HA with methacrylic anhydride, first introduced in 1999 by Smeds et al., is another method to form an MeHA [80]. The networks of hydrogels formed from the MeHA macromer can be modulated by the concentration of the MeHA macromer, the number of reactive groups, and the molecular weight of HA [81]. Poldervaart et al. synthesized an MeHA hydrogel by methacrylic anhydride for 3D printable bone scaffold fabrication. The MeHA hydrogel exhibited good biocompatibility, excellent printability, and intrinsic in vitro osteogenicity [79] (Figure 4c). In another study, Ondeck et al. demonstrated that the mechanical property of MeHA hydrogels could be modulated by monomer concentration, duration of UV exposure, and methacrylate functionalization [82]. 
(a)

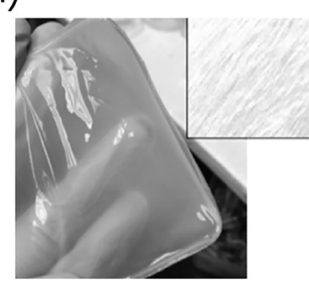

(c)
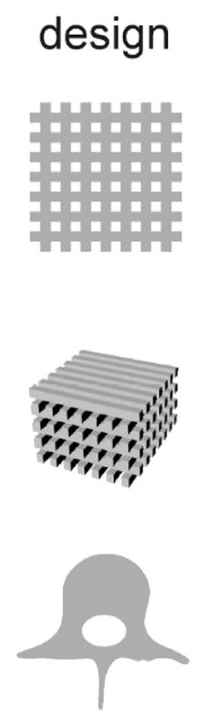

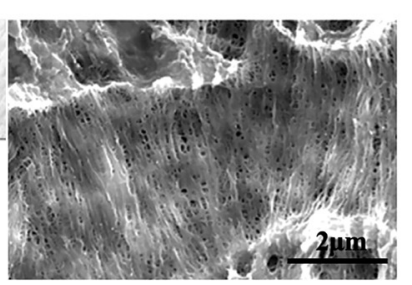

(b)

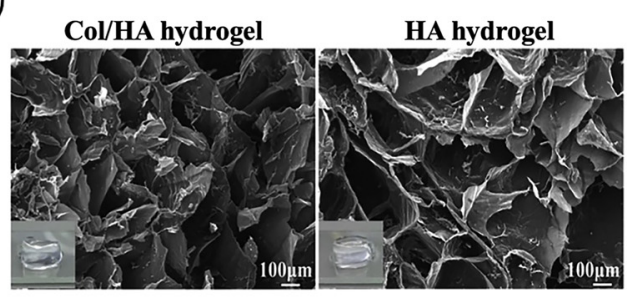

MeHA polymer in hydrogel ( \% )

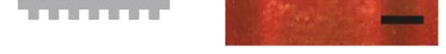

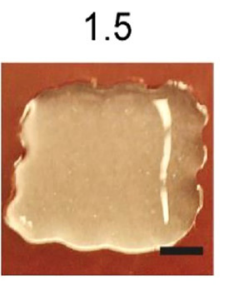

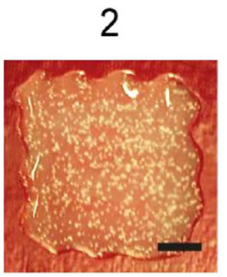

2.5
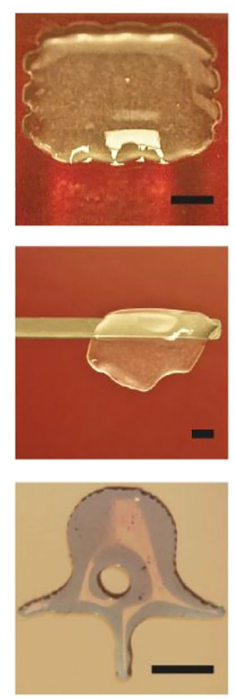
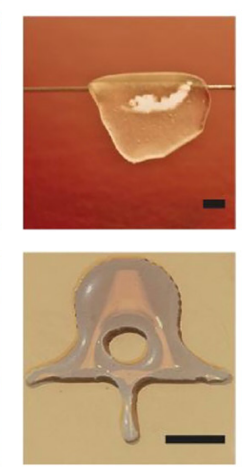
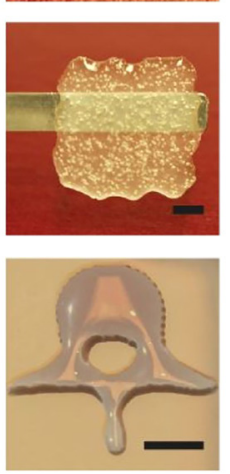
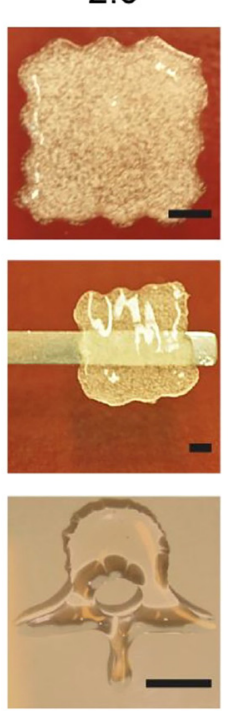

3
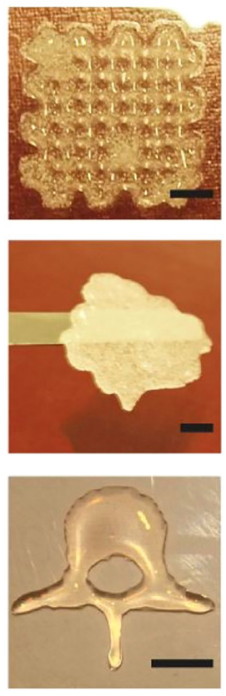

Figure 4: The GMHA showed a good printability and ability of anti-adhesion. (a) The hydrogel film composed of alginate and GMHA [74]. (b) GMHA hydrogel [76]. (c) The printability of MeHA [79].

Moreover, Zhang et al. found that MeHA hydrogels combined with adhesive peptide could enhance the proliferation and differentiation of encapsulated hiPSCs [83]. Additionally, the degree of methacrylation of HA by methacrylic anhydride can also regulate the encapsulated cell interactions. Therefore, it is crucial to evaluate the degree of methacrylation. Yousefi et al. demonstrated that infrared spectroscopy in attenuated total reflection mode could be an alternative method to evaluate the degree of methacrylation of HA hydrogels, including during the reaction progression [84].

\section{Fabrication techniques of HA-based biomaterials}

Currently, various processing techniques, such as phase separation, freeze drying, salt leaching, electrospinning, and 3D printing, have been applied for the fabrication of bone tissue-engineered constructs [21,22]. Bone tissue can be divided into compact bone tissue and spongy bone tissue. Additionally, the bone tissue in different parts has different shapes, mechanical properties, and microstructures. The biological characteristics of HA-based biomaterials were found to vary with porosity, interconnected network, and component composition. By combining with different processing techniques, HA-based biomaterials can be constructed with complex structures and various shapes for bone tissue regeneration. Therefore, this section will focus on various processing techniques used in the fabrication of HA-based biomaterials.

\subsection{Freeze drying fabrication of porous scaffolds}

Freeze drying, also known as lyophilization, is a dehydration process at a low temperature [85]. The process of 
freeze drying includes freezing scaffolds, lowering pressure, and removing the ice by sublimation. The scaffolds can turn into porous constructs with interconnected micropore structure after freeze drying. Moreover, the porosities of scaffolds can be modulated via the rate of freezing. The constructs can keep their original shape and size during the process of freeze drying. Singh et al. synthesized a three-dimensional gelatin-hyaluronic acid-alginate (GHA) polymeric scaffolds by freeze drying for bone regeneration [86]. The results demonstrated that scaffolds had a high porosity, a rapid swelling behavior, and an interconnected pore morphology (Figure 5a). Additionally, porous scaffolds enhanced cell proliferation and induced osteogenic differentiation without external growth factors. Kaczmarek et al. developed 3D porous constructs based on chitosan, collagen, and HA by freeze drying, and then used them as matrixes for the calcium phosphate in situ precipitation for bone regeneration [87] (Figure 5b). Hu et al. developed biomimetic hybrid scaffold composed of HA, chondroitin sulfate, chitosan, and nano hydroxyapatite by freeze drying technology [88] (Figure 5c). The results showed that hybrid scaffold had hierarchical micro/nano structures and enhanced the proliferation and differentiation of osteoblasts. In another study, Li et al. fabricated chitosan-HA scaffolds containing nano-pearl powder by freeze drying for bone regeneration [89] (Figure 5d). The scaffold exhibits a high porosity. In another study, freeze drying technique was used to construct a tissue-engineered scaffold composed of chitosan, collagen, and HA supplemented with nanohydroxyapatite for bone regeneration [90]. The in vivo results showed satisfactory tissue response on the implanted scaffolds.

\subsection{Salt leaching fabrication of porous scaffolds}

The salt leaching technique is a common method to fabricate porous scaffolds in the field of tissue engineering. The salt leaching technique involves mixing a polymer solution with solid salt particles of a definite diameter. The salt particles were leached after soaking in water, resulting in porous tissue-engineered scaffolds with high porosity [91]. Palumbo et al. utilized a hydrophobic/aminofunctionalized derivative of HA to fabricate a porous scaffold by salt leaching technique [92]. The porosity of scaffolds can be modulated by altering the diameter of salt particles. However, salt leaching technology has the (a)

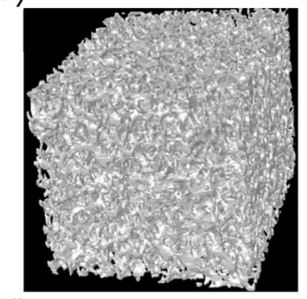

(b)

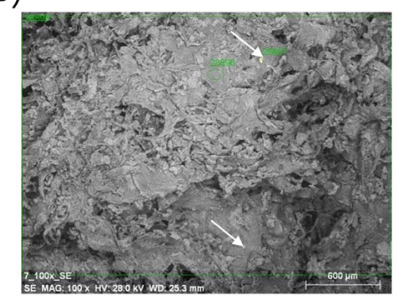

(c)

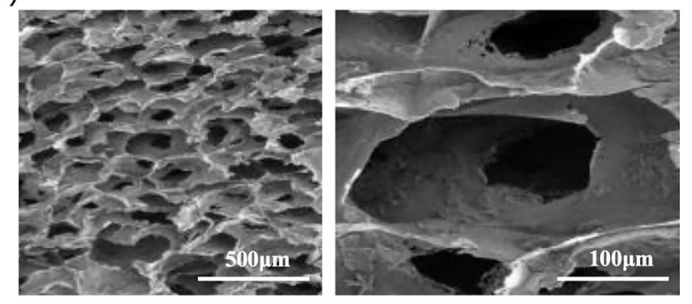

(d)

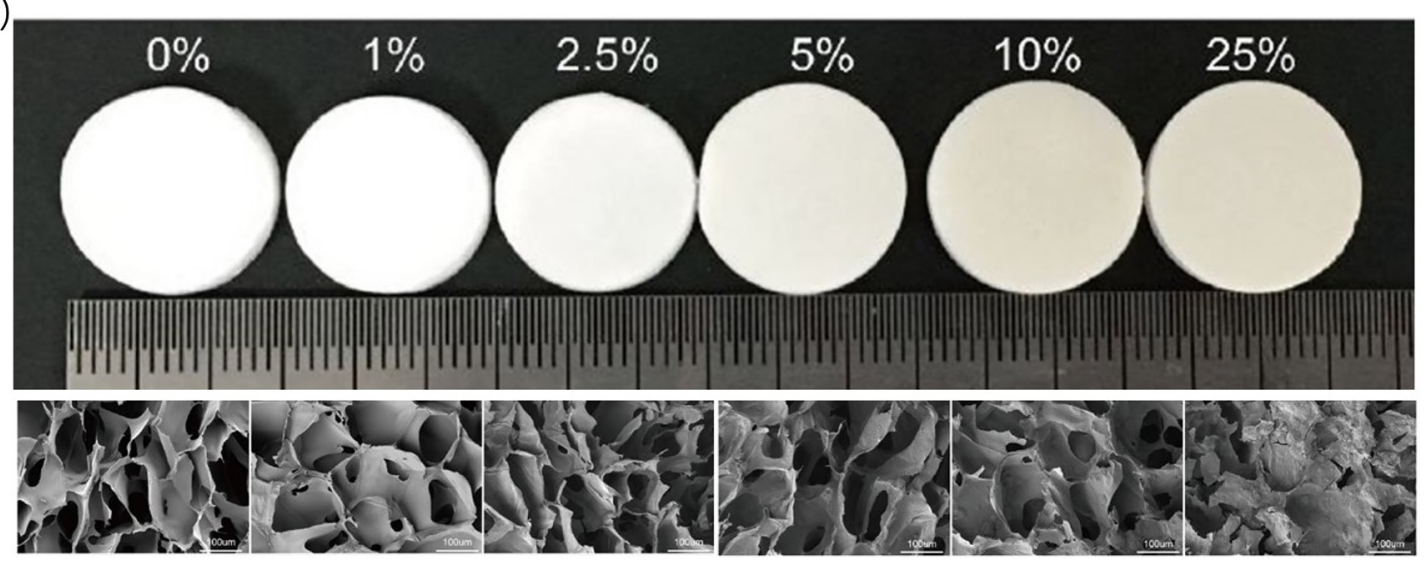

Figure 5: Various HA-based scaffolds can be constructed through freeze drying. (a) The 3D reconstruction image of GHA polymeric scaffolds [86]. (b) The SEM images of chitosan/collagen/HA scaffolds with precipitated calcium phosphate [87]. (c) The SEM images of HA/chondroitin sulfate/chitosan/nano hydroxyapatite hybrid scaffold [88]. (d) The photograph of nano-pearl powder/chitosan/HA scaffolds with different proportions of nano-pearl powder [89]. 
disadvantages of poor interconnection and abnormal pore shape.

\subsection{Phase separation fabrication of porous scaffolds}

The thermally induced phase separation (TIPS) technique is the creation of two distinct phases from a single homogeneous mixture [93]. TIPS technique is the most commonly used phase separation technology and involves the utilization of a solvent with a low melting point. Following cooling below the solvent melting point and subsequent removal of crystals by sublimation, a porous tissue-engineered scaffold is obtained [94]. TIPS technique has been widely used in recent years because of its potential to produce highly porous scaffolds with interconnected pore morphology. Erickson et al. fabricated bilayer chitosan-HA scaffolds using TIPS [95]. The results showed that the bilayer scaffold can promote the cell proliferation and migration of osteoblast-like cells. Additionally, the porosity and mechanical properties can be modulated by altering polymer solution concentration, quenching rate, and quenching temperature [96]. In another study, Jensen et al. fabricated a tissue-engineered scaffold consisting of polycaprolactone, HA, and $\beta$-TCP by TIPS [97]. The results showed that the scaffold could enhance cell migration and osteogenic differentiation in vitro.

\subsection{Electrospinning fabrication of porous scaffolds}

Electrospinning, a spinning technique, has been used for many years to generate nanofibers by utilizing electric force to draw charged threads of polymer solutions. Electrospinning originated from the textile industry [98]. Over the years, electrospinning has been gradually applied to the construction of tissue-engineered fibrous scaffolds which can mimic both the natural form and function of the ECM. Additionally, the characteristics of fibrous scaffolds can be modulated by varying the processing parameters of electrospinning [99]. Electrospinning can be used to fabricate nanometer-sized materials with the complex inner structure to mimic different types of ECMs. As one of the natural polymers, HA and HA derivatives are good candidates for electrospinning due to their biocompatibility and unique biological functions. However, HA alone cannot be used for electrospinning owing to its high viscosity at very low polymer concentration. Therefore, in most studies, HA is mixed with other polymers for electrospinning. Sujana et al. fabricated a biocompatible poly(L-lactic acid)-copoly( $\varepsilon$-caprolactone)-silk fibroin-hydroxyapatite-HA nanofibrous scaffold by electrospinning [100] (Figure 6a). The results demonstrated that nanofibrous scaffolds could enhance osteoblast proliferation, osteogenic differentiation, and mineralization. Li et al. utilized HA oligosaccharides and collagen to fabricate a biomimetic mineralized nanofiber network which could enhance cell proliferation and induce osteogenesis in vitro [101] (Figure 6b).

\subsection{D printing fabrication of porous scaffolds}

3D printing technology is the fabrication of a threedimensional object, typically layer by layer, from a computer-aided design model or other geometrical data, such as X-ray imaging, magnetic resonance imaging, ultrasound imaging techniques, and computerized tomography scan [102-104]. 3D bioprinting involves the utilization of 3D printing-like techniques to combine cells and other bioactive molecules to fabricate biomimetic tissue constructs complex 3D architecture [43,105,106]. As one of the latest biotechnologies, 3D bioprinting has been widely used in bone tissue regeneration to fabricate bone tissue-engineered constructs with geometrically defined structures for personalized patient-specific therapy. 3D bioprinting technology has the advantages of precise spatiotemporal control on the distribution of cells, small molecules, osteogenesis-related growth factors, miRNA, and osteoinductive drugs [107]. Moreover, in the process of 3D bioprinting, bioinks play a crucial role in providing a stable 3D architecture for cells and mimicking the tissue niche in situ [108]. HA and HA derivatives can be used as bioinks for 3D bioprinting due to their biocompatibility and ability to regulate encapsulated cell behaviors.

Currently, several studies have used HA or HA derivatives as bioinks for the fabrication of tissue-engineered bone constructs. Noh et al. synthesized a hybrid hydrogel from HA, hydroxyethyl acrylate (HEA), and gelatin-methacryloyl and used it as a cell-laden bioink for bone regeneration [109]. The results demonstrated that the hybrid hydrogel showed excellent biocompatibility and good printability (Figure 7a). In another study, Wei et al. developed a composite bioink consisting of HA, silk fibroin, gelatin, and tricalcium phosphate and fabricated tissue-engineered 

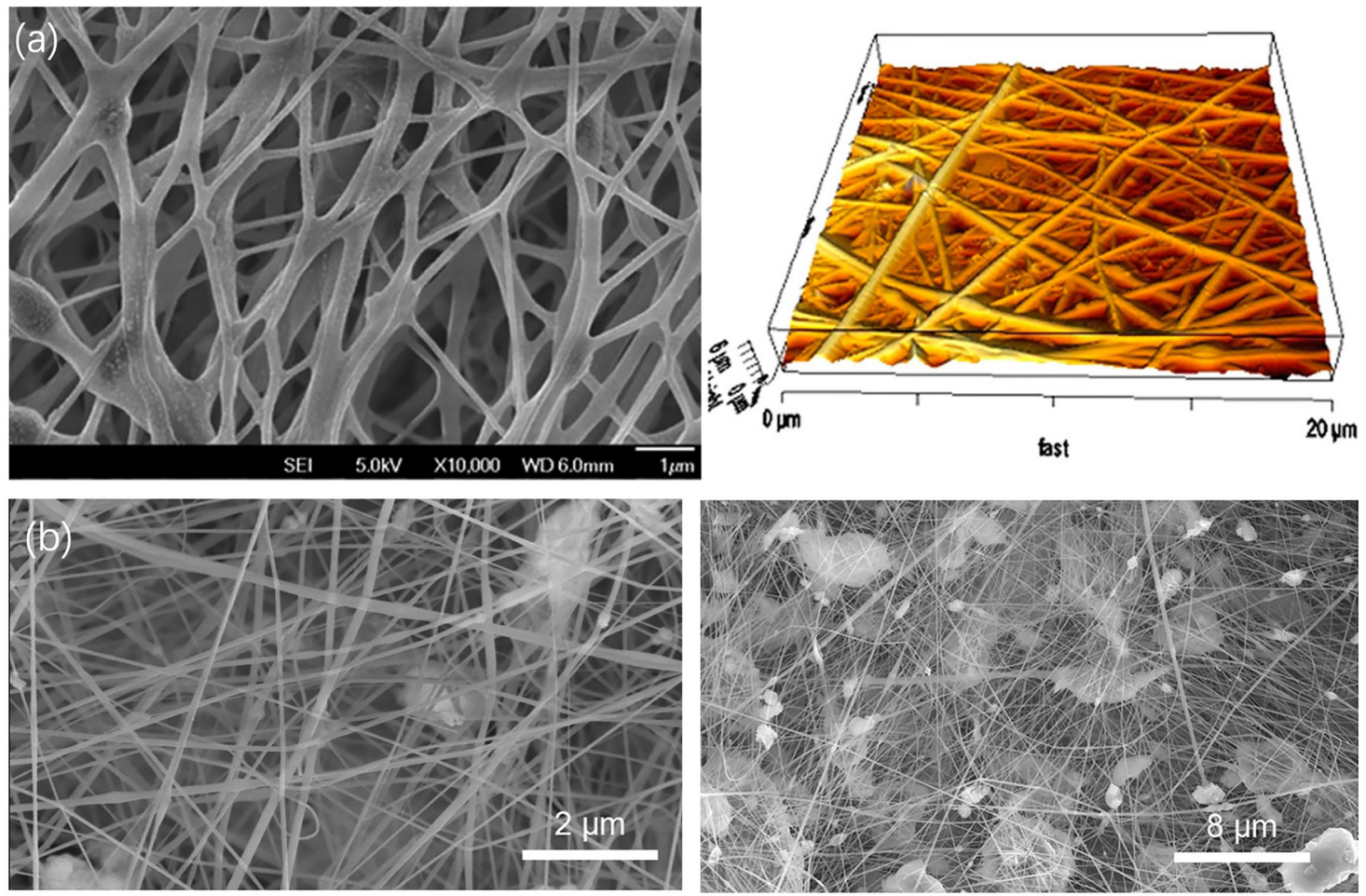

Figure 6: Various HA-based scaffolds constructed by electrospinning. (a) The SEM and 3D AFM of poly(L-lactic acid)-co-poly( $\varepsilon$-caprolactone)-silk fibroin-hydroxyapatite-HA nanofibrous scaffolds [100]. (b) The SEM images of nanofibers composed of HA oligosaccharides and collagen [101].

(a)
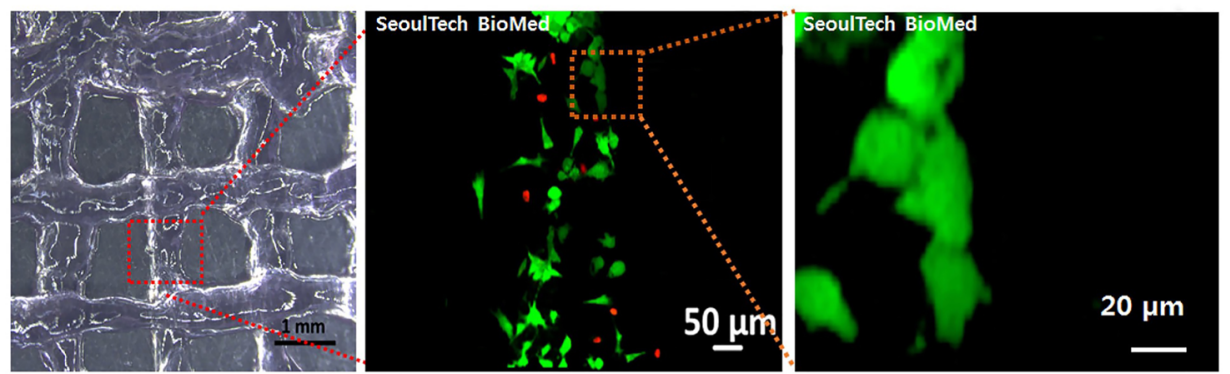

(b)

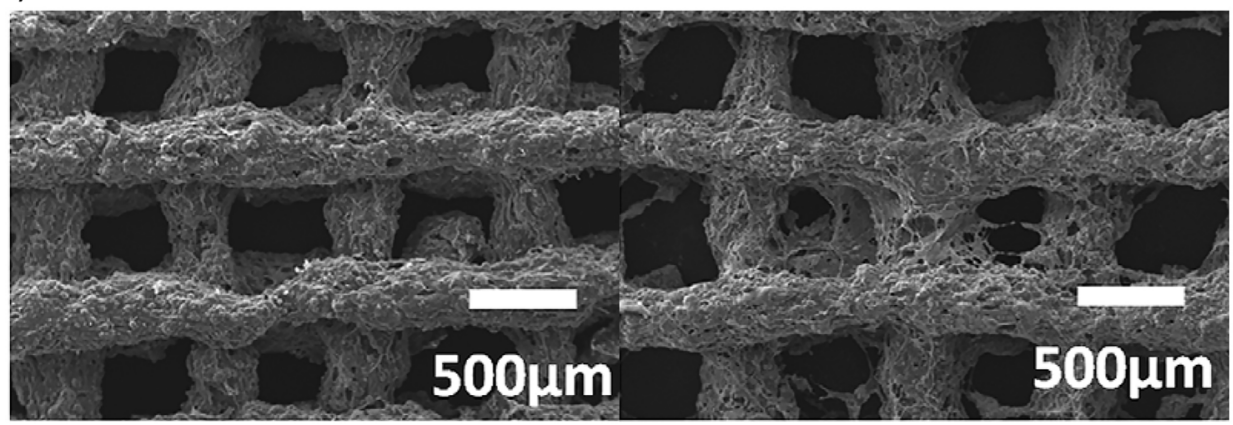

Figure 7: (a) Live and dead assay results of hybrid hydrogel by HA, HEA, and gelatin-methacryloyl [109]. (b) A bioink consisting of HA, silk fibroin, gelatin, and tricalcium phosphate treated with/without the platelet-rich plasma [110]. The HA-based bioinks showed excellent biocompatibility and good printability. 
bone constructs coated by human platelet-rich plasma (Figure 7b) [110]. Müller et al. developed a hybrid bioink for bone tissue engineering, consisting of HA methacrylate, hydroxyapatite particles, and gelatin gum methacrylate. The results found that bioink displayed a strong interlayer adhesion between two printed ink layers. In another study, Wenz et al. utilized the same method to synthesize a hydrogel to encapsulate primary human adipose-derived stem cells for tissue-engineered bone fabrication using 3D microextrusion bioprinting technology [111]. The results showed that bioink enhanced bone matrix development and remodeling. However, the structure of tissue-engineered bone construct bioprinted with HA in current studies is relatively simple, and no studies have yet to report osteogenic growth factors being incorporated in HA-derived bioinks during the process of 3D bioprinting.

\section{Applications of HA in bone tissue engineering}

\subsection{HA surface coatings for bone tissue engineering scaffolds}

In recent years, coating design on various biomaterials has been the focus in the field of biomaterial fabrication. $\mathrm{HA}$ and its derivatives have been widely applied in coating fabrication of tissue-engineered bone scaffolds, such as metallic, polymeric, or ceramic surfaces. During the process of bone repair, various coatings on the tissueengineered bone scaffolds are designed to induce favorable cell and tissue responses between the implants and surrounding host tissue [112]. As a natural ECM component, the specific biophysical properties of HA distinguish it from other components of ECM. Many technologies such as plasma spray, electrochemical technique, or polyelectrolyte multilayers are used to construct surface coating of tissue-engineered bone scaffolds [113].

\subsubsection{Osteogenic functional coatings}

Currently, many dental or orthopedic implants are made of alloys or ceramic owing to their excellent corrosion resistance and good hard-tissue compatibility [114]. However, poor osseointegration between implants and surrounding bone tissue can result in implants or prosthesis loosening [115]. The coating of dental or orthopedic implants can enhance the contact between host bone tissue and implants, reducing the risk of implant loosening and adverse reactions [116]. As one of the important components of the extracellular bone matrix, HA can promote the osseointegration of metallic and hydroxyapatite implants by modulating cell migration, adhesion, and differentiation. Song et al. developed $\mathrm{HA} /$ chitosan multilayer loaded with icariin onto the surface of the titanium implant via a layer-by-layer self-assembly system [117]. The results showed that the coating enhanced the proliferation, viability, and adhesion of osteoblasts and also promoted early osseointegration in vivo (Figure 8). Mathews et al. fabricated a tripolymer coating with chitosan, collagen type 1, and HA [118]. The results showed that the tripolymer coating enhanced mineralization and osteoblast differentiation. Moreover, HA-based coatings can be functionalized by the incorporation of growth factors and drugs. In another study, Aebli et al. fabricated an HA coating loaded with BMP-2 onto the surface of hydroxyapatite implants to promote bone formation around the implants [119].

\subsubsection{Antibacterial functional surface coatings}

Bacterial contamination at implant insertion can also result in dental implant failure [120]. However, treatment for infections around implants remains a challenge for dentists. The gold standard treatment for infection around implants is anti-infective therapy combined with implant replacement [121]. Therefore, antibacterial adhesion to the surface of the implants and reducing the risk of infection around the implants are of interest in the field of biomaterials. Currently, HA coating has also been used as a local antimicrobial or anti-adhesive barrier of implants. Valverde et al. fabricated multilayer antibacterial coatings as reservoirs of antibacterial agents onto modified titanium surfaces by using HA and chitosan [122]. The results showed that multilayer antibacterial coatings exhibited a good antibacterial property. Guarise et al. fabricated dopamine-functionalized sulfated HA coating for titanium implants [123]. The results showed that dopamine-functionalized sulfated HA coating could prevent biofilm formation and enhance osseointegration.

\subsection{Osteogenic activity carriers}

\subsubsection{Carrying osteogenesis-related cells}

Bone regeneration is a dynamic process that involves various cells, such as osteoblasts, MSCs, and ECs. MSCs 

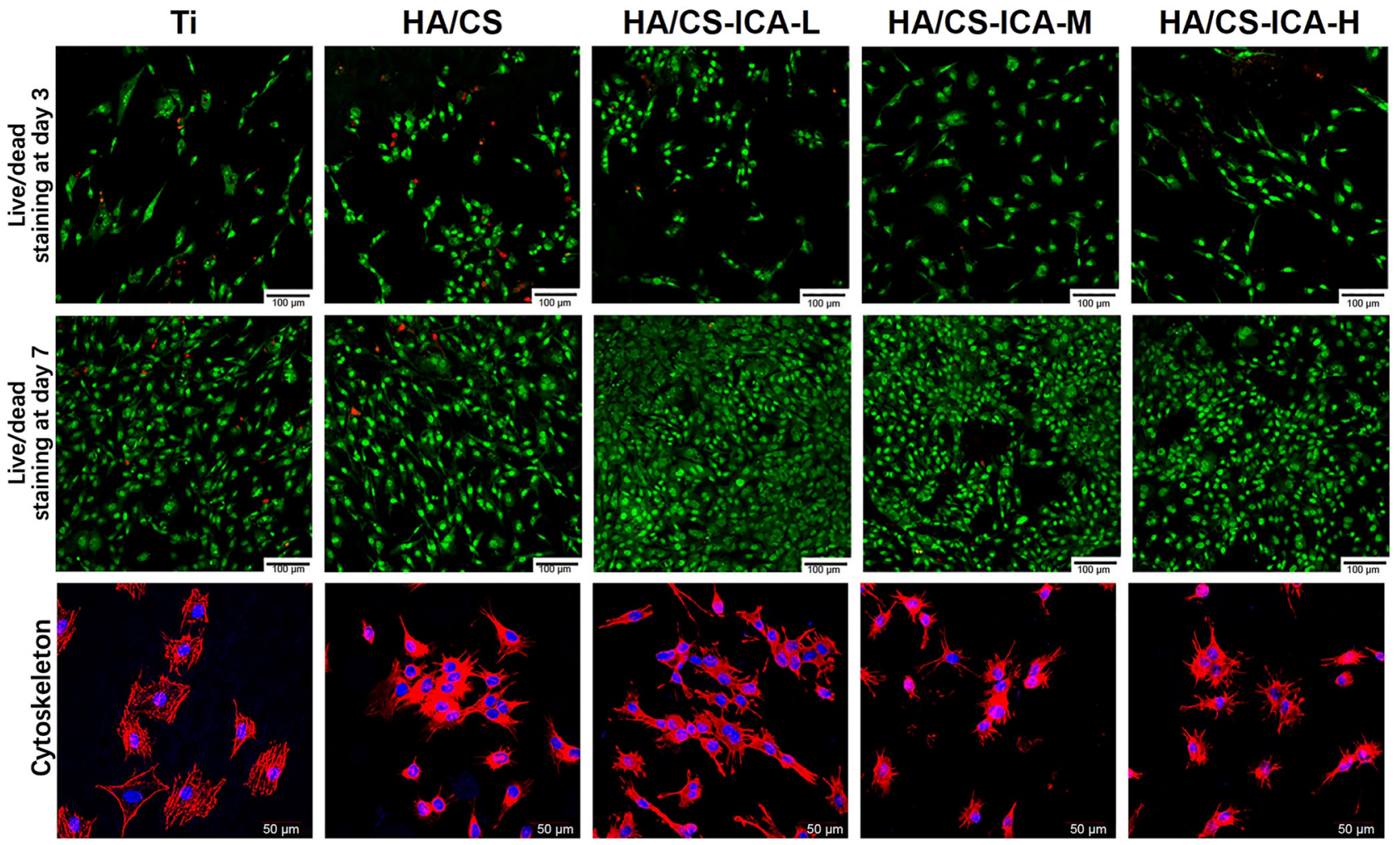

Figure 8: Live/dead and cytoskeleton staining of MC3T3-E1 cells on different surfaces of HA/chitosan multilayer coating [117].

are the most widely used type of osteoblasts in bone regeneration. MSCs come from a wide range of tissues, such as bone marrow, adipose, and umbilical cord [124]. Various HAbased hydrogels have been fabricated for cell delivery, owing to their biocompatibility and biodegradability $[125,126]$. Currently, many researchers utilize injectable HA-based hydrogels to encapsulate cells for bone regeneration by the minimally invasive method of injection. Rezaeeyazdi et al. developed an injectable hybrid hydrogel consisting of HA and gelatin [127]. The results showed that the hybrid hydrogel provided a suitable environment for cell attachment, spreading, and survival for osteoblasts (Figure 9a). In another study, Liao et al. fabricated a biocompatible thermo-gelling hydrogel scaffold, HA-g-chitosan-g-poly $(N$ isopropylacrylamide), to encapsulate stem cells for bone regeneration [128]. Moreover, HA-based hydrogels can also be used for multicell type delivery. Blood vessel formation involves the migration and proliferation of ECs and plays an important role in the process of bone regeneration [129]. Kang et al. constructed a hybrid hydrogel composed of collagen and HA as cell carriers for coculture of human adipose-derived stem cells and human umbilical vein ECs [130] (Figure 9b). In another study, Wenz et al. improved vasculogenesis and bone matrix formation through the coculture of ECs and stem cells in HA-based hydrogels [131].

\subsubsection{Carrying osteogenesis-related growth factors}

Growth factors are often a key component in the field of tissue engineering. Various growth factors play an important role in the process of bone regeneration and remolding [132]. Many growth factors alone or combined with scaffolds have been used for bone regeneration, including bone morphogenetic proteins (BMPs), fibroblast growth factors, VEGF, transforming growth factor, insulin-like growth factor, PDGF, and hepatocyte growth factor. Various growth factors can modulate the proliferation, differentiation, and recruitment of osteogenic-related cells to enhance bone formation [133]. However, the growth factor-based therapies for bone regeneration are limited by dosage-related adverse effects. Moreover, the delivery system of growth factors can also affect their efficiency, causing growth factors to be intrinsically less stable than other inorganic components, such as hydroxyapatite [134]. Therefore, how to incorporate the growth factors into the scaffolds while preserving the structural and functional integrity of the growth factors is still a challenge for researchers. As one of the natural polymers, HA is versatile for incorporating growth factors by crosslinking reaction. One common use of HA hydrogels is to accomplish spatiotemporal control of growth factor 
(a)

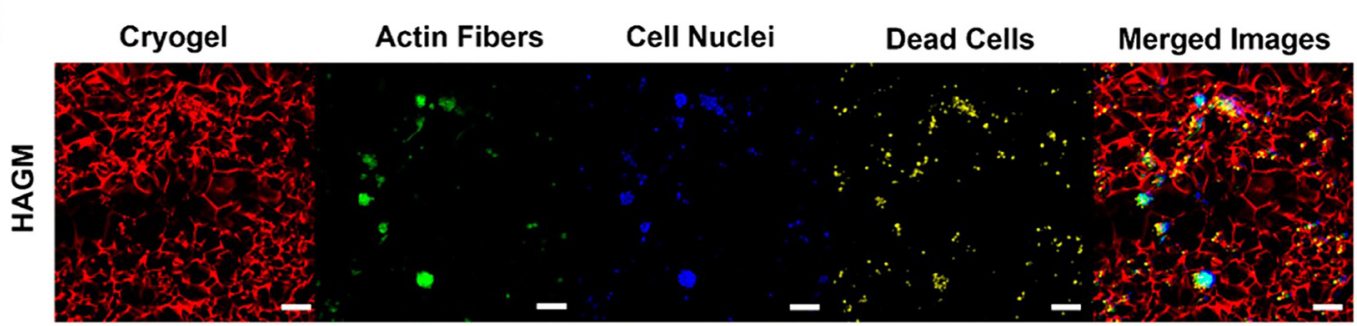

(b)
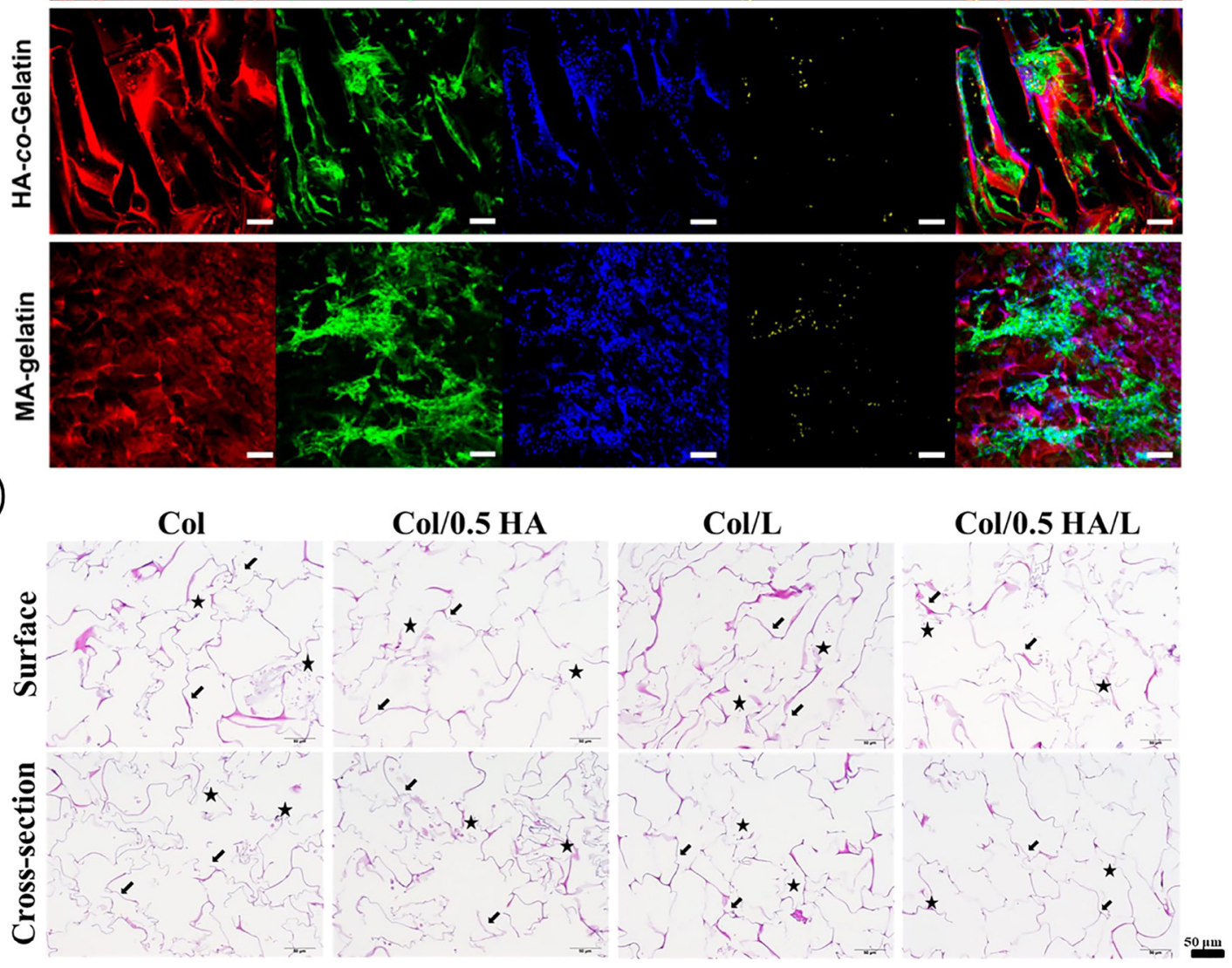

Figure 9: (a) Cell viability of hybrid hydrogel [127]; (b) hematoxylin and eosin stain of coculture of human adipose-derived stem cells and human umbilical vein ECs for 28 days in hybrid hydrogels ( $\star$ : cells, $\swarrow:$ scaffolds) [130].

release, owing to their biocompatibility, degradability, and non-immunogenicity. As a result, many researchers used HA and HA derivatives as a vehicle for growth factor delivery in bone regeneration. $\mathrm{Xu}$ et al. constructed heparin-decorated, HA-based, microscopic hydrogel particles (HGPs) for the controlled release of BMP-2 [135] (Figure 10a). The results confirmed that the addition of heparin to HGPs significantly improved the loading of BMP-2 and prolonged the release of BMP-2. In another study, Jha et al. fabricated BMP-2-loaded HA-based HGPs for growth factor's sustained release [136] (Figure 10b).

According to the types of growth factors delivered by HA and HA derivatives for bone regeneration, growth factors' delivery can be divided into single growth factor delivery and multiple growth factor delivery. Currently, most studies have used the method of single growth factor delivery to promote bone regeneration, while there are several other studies that used the combination of multiple growth factors with different functions to enhance bone formation [137-139]. Holloway et al. delivered stromal cell-derived factor- $1 \alpha$ (SDF- $1 \alpha)$ in combination with BMP2 using proteolytically degradable HA hydrogels in a critical-sized calvarial defect [140]. The results in vivo demonstrated that SDF- $1 \alpha$ and BMP-2 synergistically enhanced bone formation (Figure 10c). However, there is no consensus that multiple growth factors can enhance bone formation due to recent studies that have shown that the combination of BMP-2 and VEGF did not show a synergistic 

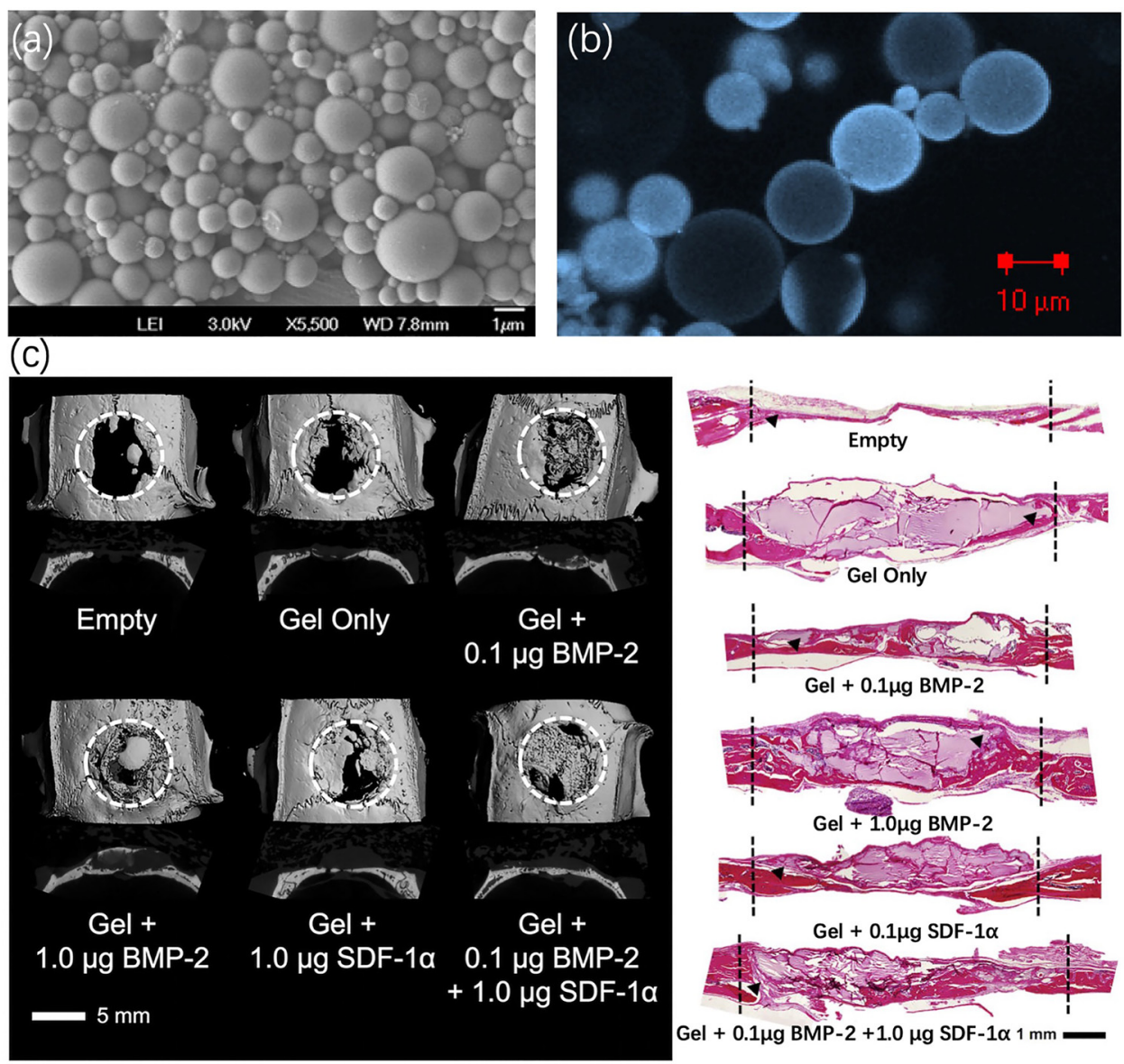

Figure 10: (a) The SEM of heparin-decorated, HA-based HGPs [135]. (b) The Cascade blue staining of BMP-2-loaded HGPs by HA [136]. (c) The $\mu \mathrm{CT}$ reconstructions and hematoxylin and eosin staining of calvarial defects for all treatment groups at 6 weeks [140].

or additive effect in a canine model of maxillary alveolar bone defects [141].

How to achieve a sustained and controlled release of growth factors is another important issue in growth factors' application for bone regeneration [133,142]. An ideal delivery system of growth factors should have advantages of adequate biodegradability, high biocompatibility, low toxicity, mechanical congruency, cost-effectiveness, ease of manufacture, and malleability [143]. In recent years, many HA-based biomaterials have been fabricated and used as delivery carriers to prolong the promotion effects of growth factors. According to the composition, all these HA-based biomaterials for growth factor delivery can be divided into single and hybrid HA-based biomaterials. For single HA-based biomaterials, the release of growth factors can be prolonged by altering the molecular weight and solution concentration of HA [144,145]. Additionally, chemical modification and crosslinking of HA in hybrid HA-based biomaterials are utilized to prolong the release period of growth factors [146,147]. HA can be combined with other components, such as chitosan, alginate, and collagen, to form hybrid HA-based biomaterials for growth factor delivery [148]. Nath et al. fabricated a hybrid hydrogel with chitosan and HA as a carrier for the controlled release of BMP-2 [149]. The results showed that hybrid hydrogel achieved a sustained release of BMP-2 for more than 1 month in vitro. In another study, Chung et al. fabricated a self-assembling collagen-HA membrane to deliver BMP-2 for bone regeneration [150]. The results found that the hybrid membrane achieved the sustained release of $17 \%$ of total loaded BMP- 2 over the course of 49 days.

\subsubsection{Carrying osteogenesis-related drugs and other components}

A variety of osteogenesis-inductive drugs have also been delivered by using HA-based biomaterials. Lee et al. developed hollow microparticles composed of catecholmodified HA shell and silica core for drug carriers [151]. 
Bae et al. fabricated photo-cured HA hydrogels loaded with simvastatin for bone regeneration [152]. In another study, Schmidt et al. demonstrated that sulfated HA and dexamethasone possess a synergistic potential in the osteogenic differentiation of MSCs [153]. Moreover, various anti-osteoporosis drugs, such as risedronate, bisphosphonate, and zoledronate, have also been incorporated into HA-based biomaterials to enhance bone regeneration and peri-implant bone augmentation [154-156]. In addition to drug delivery, several studies have shown the potential of HA-based biomaterials for the delivery of inorganic osteogenesis-inductive components, such as hydroxyapatite, beta-tricalcium phosphate, and bioactive glass $[157,158]$.

\section{$6 \mathrm{HA}$ in bone regeneration nanomedicine}

Nanomedicine is the medical application of nanotechnology. Currently, various tissue-engineered biomaterials with nanoscales such as nanoparticles and nanofibers have been fabricated by nanotechnology for bone regeneration [159]. Due to their biodegradability, biocompatibility, non-immunogenicity, and non-thrombogenicity, HA and its derivatives have been utilized to fabricate HA-based biomaterials with nanoscales for bone regeneration. Séon-Lutz et al. utilized the electrospinning technique to fabricate biomimetic nanofibrous scaffolds consisting of poly(vinyl alcohol) and HA [160]. In another study, Fischer et al. utilized collagen and HA to develop a nanofiber mesh by electrospinning for bone regeneration [161]. However, HA-based nanofibrous scaffolds constructed by electrospinning technology usually lack a large volume, which greatly limits its application for bone regeneration in vivo. Additionally, various nucleic acids have also been incorporated into HA-based nanoparticles for bone regeneration [162]. In addition, many miRNAs play important roles in the proliferation and differentiation of MSCs. Wang et al. constructed hybrid nanoparticles by chitosan and HA to deliver microRNA-21, which dramatically enhanced the osteogenesis in cell sheets of BMSCs [163]. In another study, Wu et al. utilized chitosan, tripolyphosphate, and HA to fabricate nanoparticles to deliver antimiR-138, which showed a positive effect of the MSC osteogenesis [164]. Curcumin (CUR) is a bright yellow chemical produced by Curcuma longa plants and can be used in the treatment for osteoporosis. Alendronate (ALN) can also be used to treat osteoporosis caused by menopause, steroid use, or gonadal failure. Dong et al. fabricated ALN/CUR nanoparticles decorated with HA by nanotechnology [165]. The results showed that HA-ALN/CUR nanoparticles could significantly enhance the proliferation, differentiation, and mineralization of osteoblasts in vitro.

\section{Conclusion and future perspectives}

HA and its derivatives have been utilized in the fabrication of tissue-engineered bone substitute for many years, due to their outstanding biodegradability and biocompatibility properties. The purpose of this study is to report the properties, processing, and applications of various HAbased biomaterials for bone regeneration in recent studies. As a matrix component of ECM, HA can activate several signaling pathways to modulate cell behaviors. HA and its derivatives can be chemically modified in various ways to improve their mechanical properties, bioactivity, and resistance against enzymatic degradation. By combining different processing and fabrication technologies, more highly structured HA-based biomaterials with diverse physiochemical properties can be constructed and used as a hybrid scaffold to repair different parts of bone tissue and promote bone regeneration. The hybrid scaffold exhibits excellent physicochemical and biological properties during the process of bone repair. In the field of orthopedics and dental implants, HA and its derivatives can be fabricated into coatings to promote osseointegration between the prosthesis and the surrounding bone tissue and prevent bacteria adhesion and biofilm formation. Moreover, by carrying different osteoinductive components, such as cells or growth factors, the osteogenic ability of HA-based biomaterials can be enhanced.

Although various HA-based biomaterials for bone regeneration have many advantages, there are still many challenges for researchers to face. Currently, there are few studies focusing on the molecular mechanisms of $\mathrm{HA}$ and its derivatives during the process of bone repair. Although the effects of HA and its derivatives on bone regeneration have been demonstrated by many studies, the differences in osteogenic ability between different HA derivatives still need further research. There are a lot of scientific research studies on HA and its derivatives as carriers of various osteogenesis-related cells and growth factors at present, but most of them are in the stage of laboratory research. And it is expected that the next few 
years will see the practical realization of some of these ideas in clinical trials. Additionally, how to achieve sustained, controlled release of osteogenic miRNAs in HA-based carriers and the interaction between HA and encapsulated cells should also be further studied. Furthermore, the emergence and development of 3D printing technology has opened a new field of personalized HA-based biomaterial construction. With the continuous developments of tissue engineering and material fabrication technologies, more intelligent and complex HA-based biomaterials will likely become promising candidates for bone regeneration.

Acknowledgments: This work was partially supported by the National Key Research and Development Program of China (No. 2018YFC1106800 and 2018YFB1105600), National Natural Science Foundation of China (31971251 and 31870961), and Sichuan Province Science \& Technology Department Projects (2019YFH0079, 2016CZYD0004, 2019JDTD0008, 2020YFS0036, and 2020YFS0462).

Conflict of interest: The authors declare no conflict of interest regarding the publication of this paper.

\section{References}

[1] Fakhari A, Berkland C. Applications and emerging trends of hyaluronic acid in tissue engineering, as a dermal filler and in osteoarthritis treatment. Acta Biomater. 2013;9(7): 7081-92.

[2] Peng Z, Tang P, Li Z, Wu L, Xu X, Lei H, et al. Advances in biomaterials for adipose tissue reconstruction in plastic surgery. Nanotechnol Rev. 2020;9(1):385-95.

[3] Sihan G, Dongwei F, Assem U, Dejun S, Mo Z, Zheng J, et al. Applications of polymer-based nanoparticles in vaccine field. Nanotechnol Rev. 2019;8(1):143-55.

[4] Kang JH, Kim YY, Chang JY, Kho HS. Influences of hyaluronic acid on the anticandidal activities of lysozyme and the peroxidase system. Oral Dis. 2011;17(6):577-83.

[5] Gayathri K, Vadim A, Uma Maheswari K. Nanoimmunotherapy - cloaked defenders to breach the cancer fortress. Nanotechnol Rev. 2018;7(4):317-40.

[6] Burdick JA, Prestwich GD. Hyaluronic acid hydrogels for biomedical applications. Adv Mater. 2011;23(12):H41-56.

[7] Li Z, Tao L, Yinhong X, Zheng Z, Junying C. A new classification method of nanotechnology for design integration in biomaterials. Nanotechnol Rev. 2020;9(1):820-32.

[8] Xing F, Li L, Zhou C, Long C, Wu L, Lei H, et al. Regulation and directing stem cell fate by tissue engineering functional microenvironments: Scaffold physical and chemical cues. Stem Cell Int. 2019;2019:16.

[9] Chelli Sai M, Kumar BS, Sai Pavan Prashanth S, Sai Krishna S, Muthukumar VS, Venketesh S, et al. Novel leadfree biocompatible piezoelectric Hydroxyapatite (HA) - BCZT
(Ba0.85Ca0.15Zr0.1Ti0.903) nanocrystal composites for bone regeneration. Nanotechnol Rev. 2019;8(1):61-78.

[10] Roseti L, Parisi V, Petretta M, Cavallo C, Desando G, Bartolotti I, et al. Scaffolds for bone. Tissue Eng: State Art N Perspect Mater Sci Eng: C. 2017;78:1246-62.

[11] Qiong W, Wei-shou M, Yi-du Z, Han-jun G, David H. Mechanical properties of nanomaterials: a review. Nanotechnol Rev. 2020;9(1):259-73.

[12] Cheng H, Lina W, Changchun Z, Huan S, Peng G, Xiujuan X, et al. Berberine/Ag nanoparticle embedded biomimetic calcium phosphate scaffolds for enhancing antibacterial function. Nanotechnol Rev. 2020;9(1):568-79.

[13] Myeroff C, Archdeacon M. Autogenous bone graft: donor sites and techniques. J bone Jt Surg Am volume. 2011;93(23): 2227-36.

[14] Cheng H, Jianxun S, Cheng L, Lina W, Changchun Z, Xingdong Z. Synthesis of nano zirconium oxide and its application in dentistry. Nanotechnol Rev. 2019;8(1):396-404.

[15] Wei J, David H, Denvid L. Nanoengineering in biomedicine: current development and future perspectives. Nanotechnol Rev. 2020;9(1):700-15.

[16] Liu C, Huang X, Wu Y, Deng X, Liu J, Zheng Z, et al. Review on the research progress of cement-based and geopolymer materials modified by graphene and graphene oxide. Nanotechnol Rev. 2020;9(1):155-69.

[17] Silva CR, Babo PS, Gulino M, Costa L, Oliveira JM, SilvaCorreia J, et al. Injectable and tunable hyaluronic acid hydrogels releasing chemotactic and angiogenic growth factors for endodontic regeneration. Acta Biomater. 2018;77:155-71.

[18] Zhai P, Peng X, Li B, Liu Y, Sun H, Li X. The application of hyaluronic acid in bone regeneration. Int J Biol Macromolecules. 2020;151:1224-39.

[19] Itano N. Simple primary structure, complex turnover regulation and multiple roles of hyaluronan. J Biochem. 2008;144(2):131-7.

[20] Anseth KS, Burdick JA. New directions in photopolymerizable biomaterials. MRS Bull. 2002;27(2):130-6.

[21] Zhao L, Pei X, Jiang L, Hu C, Sun J, Xing F, et al. Bionic design and 3D printing of porous titanium alloy scaffolds for bone tissue repair. Compos Part B: Eng. 2019;162: 154-61.

[22] Song P, Hu C, Pei X, Sun J, Sun H, Wu L, et al. Dual modulation of crystallinity and macro-/microstructures of 3D printed porous titanium implants to enhance stability and osseointegration. J Mater Chem B. 2019;7(17):2865-77.

[23] Xu X, Jha AK, Harrington DA, Farach-Carson MC, Jia X. Hyaluronic acid-based hydrogels: from a natural polysaccharide to complex networks. Soft Matter. 2012;8(12): 3280-94.

[24] Huang G, Huang H. Application of hyaluronic acid as carriers in drug delivery. Drug Delivery. 2018;25(1):766-72.

[25] Bastow ER, Byers S, Golub SB, Clarkin CE, Pitsillides AA, Fosang AJ. Hyaluronan synthesis and degradation in cartilage and bone. Cell Mol Life Sci. 2008;65(3):395-413.

[26] Almond A, Brass A, Sheehan JK. Deducing polymeric structure from aqueous molecular dynamics simulations of oligosaccharides: predictions from simulations of hyaluronan tetrasaccharides compared with hydrodynamic and X-ray fibre diffraction data11Edited by R. Huber. J Mol Biol. 1998;284(5):1425-37. 
[27] Scott JE, Heatley F. Hyaluronan forms specific stable tertiary structures in aqueous solution: $A^{13} C$ NMR study. Proc Natl Acad Sci USA. 1999;96(9):4850-5

[28] Tan H, Gong Y, Lao L, Mao Z, Gao C. Gelatin/chitosan/ hyaluronan ternary complex scaffold containing basic fibroblast growth factor for cartilage tissue engineering. J Mater Sci: Mater Med. 2007;18(10):1961-8.

[29] David-Raoudi M, Tranchepain F, Deschrevel B, Vincent J-C, Bogdanowicz P, Boumediene K, et al. Differential effects of hyaluronan and its fragments on fibroblasts: relation to wound healing. Wound Repair Regen. 2008;16(2):274-87.

[30] Quéré R, Andradottir S, Brun ACM, Zubarev RA, Karlsson G, Olsson K, et al. High levels of the adhesion molecule CD44 on leukemic cells generate acute myeloid leukemia relapse after withdrawal of the initial transforming event. Leukemia. 2011;25(3):515-26.

[31] Bignami A, Mansour H, Dahl D. Glial hyaluronate-binding protein in Wallerian degeneration of dog spinal cord. Glia. 1989;2(5):391-5.

[32] Zhao N, Wang X, Qin L, Guo Z, Li D. Effect of molecular weight and concentration of hyaluronan on cell proliferation and osteogenic differentiation in vitro. Biochem Biophy Res Commun. 2015;465(3):569-74.

[33] Zou L, Zou X, Chen L, Li H, Mygind T, Kassem M, et al. Effect of hyaluronan on osteogenic differentiation of porcine bone marrow stromal cells in vitro. J Orthopaedic Res. 2008;26(5):713-20.

[34] Lai J-Y, Tu IH. Adhesion, phenotypic expression, and biosynthetic capacity of corneal keratocytes on surfaces coated with hyaluronic acid of different molecular weights. Acta Biomater. 2012;8(3):1068-79.

[35] Hempel U, Möller S, Noack C, Hintze V, Scharnweber D, Schnabelrauch $M$, et al. Sulfated hyaluronan/collagen I matrices enhance the osteogenic differentiation of human mesenchymal stromal cells in vitro even in the absence of dexamethasone. Acta Biomater. 2012;8(11):4064-72.

[36] Gariboldi S, Palazzo M, Zanobbio L, Selleri S, Sommariva M, Sfondrini L, et al. Low molecular weight hyaluronic acid increases the self-defense of skin epithelium by induction of $\beta$-Defensin 2 via TLR2 and TLR4. J Immunol. 2008;181(3): 2103-10.

[37] Leppilahti M, Hellström P, Tammela TLJ. Effect of diagnostic hydrodistension and four intravesical hyaluronic acid instillations on bladder ICAM-1 intensity and association of ICAM1 intensity with clinical response in patients with interstitial cystitis. Urology. 2002;60(1):46-51.

[38] Drago L, Cappelletti L, De Vecchi E, Pignataro L, Torretta S, Mattina R. Antiadhesive and antibiofilm activity of hyaluronic acid against bacteria responsible for respiratory tract infections. APMIS. 2014;122(10):1013-9.

[39] Hemshekhar M, Thushara RM, Chandranayaka S, Sherman LS, Kemparaju K, Girish KS. Emerging roles of hyaluronic acid bioscaffolds in tissue engineering and regenerative medicine. Int J Biol Macromol. 2016;86:917-28.

[40] Bourguignon LYW, Wong G, Earle CA, Xia W. Interaction of low molecular weight hyaluronan with CD44 and toll-like receptors promotes the actin filament-associated protein 110 -actin binding and MyD88-NFKB signaling leading to proinflammatory cytokine/chemokine production and breast tumor invasion. Cytoskeleton. 2011;68(12):671-93.
[41] Campo GM, Avenoso A, Campo S, D’Ascola A, Nastasi G, Calatroni A. Molecular size hyaluronan differently modulates toll-like receptor-4 in LPS-induced inflammation in mouse chondrocytes. Biochimie. 2010;92(2):204-15.

[42] Loi F, Córdova LA, Pajarinen J, Lin T-H, Yao Z, Goodman SB. Inflammation, fracture and bone repair. Bone. 2016;86: 119-30.

[43] Xing F, Xiang Z, Rommens PM, Ritz U. 3D bioprinting for vascularized tissue-engineered bone fabrication. Materials. 2020;13(10):2278.

[44] Zhang Z-Y, Teoh S-H, Chong MSK, Lee ESM, Tan L-G, Mattar CN, et al. Neo-vascularization and bone formation mediated by fetal mesenchymal stem cell tissue-engineered bone grafts in critical-size femoral defects. Biomaterials. 2010;31(4):608-20.

[45] Zhou J, Lin H, Fang T, Li X, Dai W, Uemura T, et al. The repair of large segmental bone defects in the rabbit with vascularized tissue engineered bone. Biomaterials. 2010;31(6):1171-9.

[46] Tian X, Azpurua J, Hine C, Vaidya A, Myakishev-Rempel M, Ablaeva J, et al. High-molecular-mass hyaluronan mediates the cancer resistance of the naked mole rat. Nature. 2013;499(7458):346-9.

[47] Slevin M, Krupinski J, Gaffney J, Matou S, West D, Delisser H, et al. Hyaluronan-mediated angiogenesis in vascular disease: uncovering RHAMM and CD44 receptor signaling pathways. Matrix Biol. 2007;26(1):58-68.

[48] Wang Y, Han G, Guo B, Huang J. Hyaluronan oligosaccharides promote diabetic wound healing by increasing angiogenesis. Pharmacol Rep. 2016;68(6):1126-32.

[49] Ciccone V, Zazzetta M, Morbidelli L. Comparison of the effect of two hyaluronic acid preparations on fibroblast and endothelial cell functions related to angiogenesis. Cells. 2019;8(12):1479.

[50] Matsumoto Y, Arai K, Momose H, Kuroyanagi Y. Development of a wound dressing composed of a hyaluronic acid sponge containing arginine. J Biomater Sci, Polym Ed. 2009;20(7-8): 993-1004.

[51] Hoffman AS. Hydrogels for biomedical applications. Adv Drug Deliv Rev. 2012;64:18-23.

[52] Stern R, Asari AA, Sugahara KN. Hyaluronan fragments: an information-rich system. Eur J Cell Biol. 2006;85(8):699-715.

[53] Fraser JRE, Laurent TC, Engström-Laurent A, Laurent UGB. Elimination of hyaluronic acid from the blood stream in the human. Clin Exp Pharmacol Physiol. 1984;11(1):17-25.

[54] Tiwari S, Bahadur P. Modified hyaluronic acid based materials for biomedical applications. Int J Biol Macromol. 2019;121:556-71.

[55] Chen F, Ni Y, Liu B, Zhou T, Yu C, Su Y, et al. Self-crosslinking and injectable hyaluronic acid/RGD-functionalized pectin hydrogel for cartilage tissue engineering. Carbohydr Polym. 2017;166:31-44.

[56] Wang H, Zhu D, Paul A, Cai L, Enejder A, Yang F, et al. Covalently adaptable elastin-like protein-hyaluronic acid (ELP-HA) hybrid hydrogels with secondary thermoresponsive crosslinking for injectable stem cell delivery. Adv Funct Mater. 2017;27(28):1605609.

[57] Ossipov DA, Piskounova S, Varghese OP, Hilborn J. Functionalization of hyaluronic acid with chemoselective groups via a disulfide-based protection strategy for 
in situ formation of mechanically stable hydrogels.

Biomacromolecules. 2010;11(9):2247-54.

[58] Park JK, Shim J-H, Kang KS, Yeom J, Jung HS, Kim JY, et al. Solid free-form fabrication of tissue-engineering scaffolds with a poly(lactic-co-glycolic acid) grafted hyaluronic acid conjugate encapsulating an intact bone morphogenetic protein-2/poly(ethylene glycol) complex. Adv Funct Mater. 2011;21(15):2906-12.

[59] Stichler S, Böck T, Paxton N, Bertlein S, Levato R, Schill V, et al. Double printing of hyaluronic acid/poly(glycidol) hybrid hydrogels with poly $(\varepsilon$-caprolactone) for MSC chondrogenesis. Biofabrication. 2017;9(4):044108.

[60] Hosack LW, Firpo MA, Scott JA, Prestwich GD, Peattie RA. Microvascular maturity elicited in tissue treated with cytokine-loaded hyaluronan-based hydrogels. Biomaterials. 2008;29(15):2336-47.

[61] Shu XZ, Liu Y, Palumbo F, Prestwich GD. Disulfide-crosslinked hyaluronan-gelatin hydrogel films: a covalent mimic of the extracellular matrix for in vitro cell growth. Biomaterials. 2003;24(21):3825-34.

[62] Kazemirad S, Heris HK, Mongeau L. Viscoelasticity of hyaluronic acid-gelatin hydrogels for vocal fold tissue engineering. J Biomed Mater Res Part B: Appl Biomater. 2016;104(2):283-90.

[63] Vanderhooft JL, Mann BK, Prestwich GD. Synthesis and characterization of novel thiol-reactive poly(ethylene glycol) cross-linkers for extracellular-matrix-mimetic biomaterials. Biomacromolecules. 2007;8(9):2883-9.

[64] Schmelzer E, Triolo F, Turner ME, Thompson RL, Zeilinger K, Reid LM, et al. Three-dimensional perfusion bioreactor culture supports differentiation of human fetal liver cells. Tissue Eng Part A. 2010;16(6):2007-16.

[65] Lee F, Chung JE, Kurisawa M. An injectable enzymatically crosslinked hyaluronic acid-tyramine hydrogel system with independent tuning of mechanical strength and gelation rate. Soft Matter. 2008;4(4):880-7.

[66] Kim KS, Park SJ, Yang JA, Jeon JH, Bhang SH, Kim BS, et al. Injectable hyaluronic acid-tyramine hydrogels for the treatment of rheumatoid arthritis. Acta Biomater. 2011;7(2): 666-74.

[67] Zhang Y, Chen H, Zhang T, Zan Y, Ni T, Cao Y, et al. Injectable hydrogels from enzyme-catalyzed crosslinking as BMSCsladen scaffold for bone repair and regeneration. Mater Sci Eng: C. 2019;96:841-9.

[68] Jooybar E, Abdekhodaie MJ, Alvi M, Mousavi A, Karperien M, Dijkstra PJ. An injectable platelet lysate-hyaluronic acid hydrogel supports cellular activities and induces chondrogenesis of encapsulated mesenchymal stem cells. Acta Biomater. 2019;83:233-44.

[69] Yan S, Wang T, Feng L, Zhu J, Zhang K, Chen X, et al. Injectable in situ self-cross-linking hydrogels based on poly(l-glutamic acid) and alginate for cartilage tissue engineering. Biomacromolecules. 2014;15(12):4495-508.

[70] Trombino S, Servidio C, Curcio F, Cassano R. Strategies for hyaluronic acid-based hydrogel design in drug delivery. Pharmaceutics. 2019;11(8):407.

[71] Ifkovits JL, Devlin JJ, Eng G, Martens TP, Vunjak-Novakovic G, Burdick JA. Biodegradable fibrous scaffolds with tunable properties formed from photo-cross-linkable poly(glycerol sebacate). ACS Appl Mater \& Interfaces. 2009;1(9):1878-86.
[72] Hennink WE, van Nostrum CF. Novel crosslinking methods to design hydrogels. Adv Drug Deliv Rev. 2012;64:223-36.

[73] Li X, Luan S, Shi H, Yang H, Song L, Jin J, et al. Improved biocompatibility of poly(styrene- $b$-(ethylene-co-butylene)- $b$ styrene) elastomer by a surface graft polymerization of hyaluronic acid. Colloids Surf B: Biointerfaces. 2013;102:210-7.

[74] Mayes SM, Davis J, Scott J, Aguilar V, Zawko SA, Swinnea S, et al. Polysaccharide-based films for the prevention of unwanted postoperative adhesions at biological interfaces. Acta Biomater. 2020;106:92-101.

[75] Ma X, Liu S, Tang H, Yang R, Chi B, Ye Z. In situ photocrosslinked hyaluronic acid and poly( $\gamma$-glutamic acid) hydrogels as injectable drug carriers for load-bearing tissue application. J Biomater Science, Polym Ed. 2018;29(18):2252-66.

[76] Zhang T, Chen H, Zhang Y, Zan Y, Ni T, Liu M, et al. Photocrosslinkable, bone marrow-derived mesenchymal stem cells-encapsulating hydrogel based on collagen for osteogenic differentiation. Colloids Surf B: Biointerfaces. 2019;174:528-35.

[77] Khoshakhlagh P, Moore MJ. Photoreactive interpenetrating network of hyaluronic acid and Puramatrix as a selectively tunable scaffold for neurite growth. Acta Biomater. 2015;16:23-34.

[78] Hsieh Y-H, Shen B-Y, Wang Y-H, Lin B, Lee H-M, Hsieh M-F. Healing of osteochondral defects implanted with biomimetic scaffolds of poly( $\varepsilon$-caprolactone)/hydroxyapatite and glycidyl-methacrylate-modified hyaluronic acid in a minipig. Int J Mol Sci. 2018;19(4):1125.

[79] Poldervaart MT, Goversen B, de Ruijter M, Abbadessa A, Melchels FPW, Öner FC, et al. 3D bioprinting of methacrylated hyaluronic acid (MeHA) hydrogel with intrinsic osteogenicity. Plos One. 2017;12(6):e0177628.

[80] Smeds KA, Pfister-Serres A, Hatchell DL, Grinstaff MW. Synthesis of a novel polysaccharide hydrogel. J Macromol Sci, Part A. 1999;36(7-8):981-9.

[81] Burdick JA, Chung C, Jia X, Randolph MA, Langer R. Controlled degradation and mechanical behavior of photopolymerized hyaluronic acid networks. Biomacromolecules. 2005;6(1):386-91.

[82] Ondeck MG, Engler AJ. Mechanical characterization of a dynamic and tunable methacrylated hyaluronic acid hydrogel. J Biomech Eng. 2016;138:2.

[83] Zhang X, Zhou P, Zhao Y, Wang M, Wei S. Peptide-conjugated hyaluronic acid surface for the culture of human induced pluripotent stem cells under defined conditions. Carbohydr Polym. 2016;136:1061-4.

[84] Yousefi F, Kandel S, Pleshko N. Infrared spectroscopic quantification of methacrylation of hyaluronic acid: a scaffold for tissue engineering applications. Appl Spectrosc. 2018;72(10):1455-66.

[85] Chen J, Peng C, Nie J, Kennedy JF, Ma G. Lyophilization as a novel approach for preparation of water resistant $\mathrm{HA}$ fiber membranes by crosslinked with EDC. Carbohydr Polym. 2014;102:8-11.

[86] Singh D, Tripathi A, Zo S, Singh D, Han SS. Synthesis of composite gelatin-hyaluronic acid-alginate porous scaffold and evaluation for in vitro stem cell growth and in vivo tissue integration. Colloids Surf B: Biointerfaces. 2014;116:502-9.

[87] Kaczmarek B, Sionkowska A, Kozlowska J, Osyczka AM. New composite materials prepared by calcium phosphate 
precipitation in chitosan/collagen/hyaluronic acid sponge cross-linked by EDC/NHS. Int J Biol Macromolecules. 2018;107:247-53.

[88] Hu Y, Chen J, Fan T, Zhang Y, Zhao Y, Shi X, et al. Biomimetic mineralized hierarchical hybrid scaffolds based on in situ synthesis of nano-hydroxyapatite/chitosan/chondroitin sulfate/hyaluronic acid for bone tissue engineering. Colloids Surf B: Biointerfaces. 2017;157:93-100.

[89] Li X, Xu P, Cheng Y, Zhang W, Zheng B, Wang Q. Nano-pearl powder/chitosan-hyaluronic acid porous composite scaffold and preliminary study of its osteogenesis mechanism. Mater Sci Eng: C. 2020;111:110749.

[90] Kaczmarek B, Sionkowska A, Gołyńska M, Polkowska I, Szponder T, Nehrbass D, et al. In vivo study on scaffolds based on chitosan, collagen, and hyaluronic acid with hydroxyapatite. Int J Biol Macromol. 2018;118:938-44.

[91] Laschke MW, Strohe A, Menger MD, Alini M, Eglin D. In vitro and in vivo evaluation of a novel nanosize hydroxyapatite particles/poly(ester-urethane) composite scaffold for bone tissue engineering. Acta Biomater. 2010;6(6):2020-7.

[92] Palumbo FS, Agnello S, Fiorica C, Pitarresi G, Puleio R, Tamburello A, et al. Hyaluronic acid derivative with improved versatility for processing and biological functionalization. Macromol Biosci. 2016;16(10):1485-96.

[93] Collins MN, Birkinshaw C. Hyaluronic acid based scaffolds for tissue engineering - a review. Carbohydr Polym. 2013;92(2): 1262-79.

[94] De France KJ, Xu F, Hoare T. Structured macroporous hydrogels: progress, challenges, and opportunities. advanced healthcare. Materials. 2018;7(1):1700927.

[95] Erickson AE, Sun J, Lan Levengood SK, Swanson S, Chang F-C, Tsao CT, et al. Chitosan-based composite bilayer scaffold as an in vitro osteochondral defect regeneration model. Biomed Microdevices. 2019;21(2):34.

[96] Raeisdasteh Hokmabad V, Davaran S, Ramazani A, Salehi R. Design and fabrication of porous biodegradable scaffolds: a strategy for tissue engineering. J Biomater Science, Polym Ed. 2017;28(16):1797-825.

[97] Jensen J, Kraft DCE, Lysdahl H, Foldager CB, Chen $M$, Kristiansen $A A$, et al. Functionalization of polycaprolactone scaffolds with hyaluronic acid and $\beta$-TCP facilitates migration and osteogenic differentiation of human dental pulp stem cells in vitro. Tissue Eng Part A. 2014;21(3-4):729-39.

[98] Subbiah T, Bhat GS, Tock RW, Parameswaran S, Ramkumar SS. Electrospinning of nanofibers. J Appl Polym Sci. 2005;96(2):557-69.

[99] Qasim SB, Najeeb S, Delaine-Smith RM, Rawlinson A, Ur, Rehman I. Potential of electrospun chitosan fibers as a surface layer in functionally graded GTR membrane for periodontal regeneration. Dental Mater. 2017;33(1):71-83.

[100] Sujana A, Venugopal JR, Velmurugan B, Góra A, Salla M, Ramakrishna S. Hydroxyapatite-intertwined hybrid nanofibres for the mineralization of osteoblasts. J Tissue Eng Regenerative Med. 2017;11(6):1853-64.

[101] Li M, Zhang X, Jia W, Wang Q, Liu Y, Wang X, et al. Improving in vitro biocompatibility on biomimetic mineralized collagen bone materials modified with hyaluronic acid oligosaccharide. Mater Sci Eng: C. 2019;104:110008.

[102] Gopinathan J, Noh I. Recent trends in bioinks for 3D printing. Biomater Res. 2018;22:11.
[103] Pei X, Ma L, Zhang B, Sun J, Sun Y, Fan Y, et al. Creating hierarchical porosity hydroxyapatite scaffolds with osteoinduction by three-dimensional printing and microwave sintering. Biofabrication. 2017;9(4):045008.

[104] Zhang B, Pei X, Zhou C, Fan Y, Jiang Q, Ronca A, et al. The biomimetic design and 3D printing of customized mechanical properties porous Ti6Al4V scaffold for load-bearing bone reconstruction. Mater Des. 2018;152:30-9.

[105] Zhang B, Pei X, Song P, Sun H, Li H, Fan Y, et al. Porous bioceramics produced by inkjet 3D printing: effect of printing ink formulation on the ceramic macro and micro porous architectures control. Compos Part B: Eng. 2018;155:112-21.

[106] Hu X, Yang Z, Kang S, Jiang M, Zhou Z, Gou J, et al. Cellulose hydrogel skeleton by extrusion 3D printing of solution. Nanotechnol Rev. 2020;9(1):345-53.

[107] Zhang B, Sun H, Wu L, Ma L, Xing F, Kong Q, et al. 3D printing of calcium phosphate bioceramic with tailored biodegradation rate for skull bone tissue reconstruction. Bio-Design Manuf. 2019;2(3):161-71.

[108] Anada T, Pan C-C, Stahl AM, Mori S, Fukuda J, Suzuki O, et al. Vascularized bone-mimetic hydrogel constructs by 3D bioprinting to promote osteogenesis and angiogenesis. Int J Mol Sci. 2019;20(5):1096.

[109] Noh I, Kim N, Tran HN, Lee J, Lee C. 3D printable hyaluronic acid-based hydrogel for its potential application as a bioink in tissue engineering. Biomater Res. 2019;23(1):3.

[110] Wei L, Wu S, Kuss M, Jiang X, Sun R, Reid P, et al. 3D printing of silk fibroin-based hybrid scaffold treated with platelet rich plasma for bone tissue engineering. Bioact Mater. 2019;4: 256-60.

[111] Wenz A, Borchers K, Tovar GEM, Kluger PJ. Bone matrix production in hydroxyapatite-modified hydrogels suitable for bone bioprinting. Biofabrication. 2017;9(4):044103.

[112] Knopf-Marques H, Pravda M, Wolfova L, Velebny V, Schaaf P, Vrana NE, et al. Hyaluronic acid and its derivatives in coating and delivery systems: applications in tissue engineering, regenerative medicine and immunomodulation. Adv Healthcare Mater. 2016;5(22):2841-55.

[113] Hanawa T. Titanium-tissue interface reaction and its control with surface treatment. Front Bioeng Biotechnol. 2019;7:170.

[114] Rupp F, Liang L, Geis-Gerstorfer J, Scheideler L, Hüttig F. Surface characteristics of dental implants: a review. Dental Mater. 2018;34(1):40-57.

[115] Liaw K, Delfini RH, Abrahams JJ. Dental implant complications. seminars in ultrasound. CT MRI. 2015;36(5):427-33.

[116] Förster Y, Rentsch C, Schneiders W, Bernhardt R, Simon JC, Worch $\mathrm{H}$, et al. Surface modification of implants in long bone. Biomatter. 2012;2(3):149-57.

[117] Song Y, Ma A, Ning J, Zhong X, Zhang Q, Zhang X, et al. Loading icariin on titanium surfaces by phase-transited lysozyme priming and layer-by-layer self-assembly of hyaluronic acid/chitosan to improve surface osteogenesis ability. Int J Nanomed. 2018;13:6751-67.

[118] Mathews S, Bhonde R, Gupta PK, Totey S. A novel tripolymer coating demonstrating the synergistic effect of chitosan, collagen type 1 and hyaluronic acid on osteogenic differentiation of human bone marrow derived mesenchymal stem cells. Biochem Biophy Res Commun. 2011;414(1):270-6.

[119] Aebli N, Stich H, Schawalder P, Theis J-C, Krebs J. Effects of bone morphogenetic protein- 2 and hyaluronic acid on the 
osseointegration of hydroxyapatite-coated implants: an experimental study in sheep. J Biomed Mater Res Part A. 2005;73A(3):295-302.

[120] Peleg O, Blinder D, Yudovich K, Yakirevitch A. Microflora of normal maxillary sinuses: does it justify perioperative antibiotic treatment in sinus augmentation procedures. Clin Oral Investig. 2019;23(5):2173-7.

[121] Davidson DJ, Spratt D, Liddle AD. Implant materials and prosthetic joint infection: the battle with the biofilm. EFORT Open Rev. 2019;4(11):633-9.

[122] Valverde A, Pérez-Álvarez L, Ruiz-Rubio L, Pacha Olivenza MA, García Blanco MB, Díaz-Fuentes M, et al. Antibacterial hyaluronic acid/chitosan multilayers onto smooth and micropatterned titanium surfaces. Carbohydr Polym. 2019;207:824-33.

[123] Guarise C, Barbera C, Pavan M, Pluda S, Celestre M, Galesso D. Dopamine-functionalized sulphated hyaluronic acid as a titanium implant coating enhances biofilm prevention and promotes osseointegration. Biofouling. 2018;34(7):719-30.

[124] Walmsley GG, Ransom RC, Zielins ER, Leavitt T, Flacco JS, $\mathrm{Hu}$ MS, et al. Stem cells in bone regeneration. Stem Cell Rev Rep. 2016;12(5):524-9.

[125] Prè ED, Conti G, Sbarbati A. Hyaluronic acid (HA) scaffolds and multipotent stromal cells (MSCs) in regenerative medicine. Stem Cell Rev Rep. 2016;12(6):664-81.

[126] Luan C, Liu P, Chen R, Chen B. Hydrogel based 3D carriers in the application of stem cell therapy by direct injection. Nanotechnol Rev. 2017;6(5):435-48.

[127] Rezaeeyazdi M, Colombani T, Memic A, Bencherif SA. Injectable hyaluronic acid-co-gelatin cryogels for tissueengineering applications. Materials. 2018;11(8):1374.

[128] Liao HT, Tsai M-J, Brahmayya M, Chen J-P. Bone regeneration using adipose-derived stem cells in injectable thermogelling hydrogel scaffold containing platelet-rich plasma and biphasic calcium phosphate. Int J Mol Sci. 2018;19(9):2537.

[129] Xia Y, Sun J, Zhao L, Zhang F, Liang X-J, Guo Y, et al. Magnetic field and nano-scaffolds with stem cells to enhance bone regeneration. Biomaterials. 2018;183:151-70.

[130] Kang PL, Huang HH, Chen T, Ju KC, Kuo SM. Angiogenesispromoting effect of LIPUS on hADSCs and HUVECs cultured on collagen/hyaluronan scaffolds. Mater Sci Eng: C. 2019;102:22-33.

[131] Wenz A, Tjoeng I, Schneider I, Kluger PJ, Borchers K. Improved vasculogenesis and bone matrix formation through coculture of endothelial cells and stem cells in tissue-specific methacryloyl gelatin-based hydrogels. Biotechnol Bioeng. 2018;115(10):2643-53.

[132] Venkatesan J, Anil S, Kim S-K, Shim MS. Chitosan as a vehicle for growth factor delivery: various preparations and their applications in bone tissue regeneration. Int J Biol Macromol. 2017;104:1383-97.

[133] Bai Y, Yin G, Huang Z, Liao X, Chen X, Yao Y, et al. Localized delivery of growth factors for angiogenesis and bone formation in tissue engineering. Int Immunopharmacol. 2013;16(2):214-23.

[134] Subbiah R, Guldberg RE. Materials science and design principles of growth factor delivery systems in tissue engineering and regenerative medicine. Adv Healthcare Mater. 2019;8(1):1801000.
[135] Xu X, Jha AK, Duncan RL, Jia X. Heparin-decorated, hyaluronic acid-based hydrogel particles for the controlled release of bone morphogenetic protein 2. Acta Biomater. 2011;7(8): 3050-9.

[136] Jha AK, Yang W, Kirn-Safran CB, Farach-Carson MC, Jia X. Perlecan domain I-conjugated, hyaluronic acid-based hydrogel particles for enhanced chondrogenic differentiation via BMP-2 release. Biomaterials. 2009;30(36): 6964-75.

[137] Häckel S, Zolfaghar M, Du J, Hoppe S, Benneker LM, Garstka N, et al. Fibrin-hyaluronic acid hydrogel (RegenoGel) with fibroblast growth factor-18 for in vitro 3D culture of human and bovine nucleus pulposus cells. Int J Mol Sci. 2019;20(20):5036.

[138] Yan HJ, Casalini T, Hulsart-Billström G, Wang S, Oommen OP, Salvalaglio M, et al. Synthetic design of growth factor se questering extracellular matrix mimetic hydrogel for promoting in vivo bone formation. Biomaterials. 2018;161: 190-202.

[139] Bae MS, Ohe J-Y, Lee JB, Heo DN, Byun W, Bae H, et al. Photocured hyaluronic acid-based hydrogels containing growth and differentiation factor 5 (GDF-5) for bone tissue regeneration. Bone. 2014;59:189-98.

[140] Holloway JL, Ma H, Rai R, Hankenson KD, Burdick JA. Synergistic effects of SDF-1 $\alpha$ and BMP-2 delivery from proteolytically degradable hyaluronic acid hydrogels for bone repair. Macromol Biosci. 2015;15(9):1218-23.

[141] Kim SK, Cho TH, Han JJ, Kim IS, Park Y, Hwang SJ. Comparative study of BMP-2 alone and combined with VEGF carried by hydrogel for maxillary alveolar bone regeneration. Tissue Eng Regenerative Med. 2016;13(2):171-81.

[142] Bayer IS. Hyaluronic acid and controlled release: a review. Molecules. 2020;25(11):2649.

[143] Zhang G, Suggs LJ. Matrices and scaffolds for drug delivery in vascular tissue engineering. Adv Drug Deliv Rev. 2007;59(4):360-73.

[144] Jha AK, Mathur A, Svedlund FL, Ye J, Yeghiazarians Y, Healy KE. Molecular weight and concentration of heparin in hyaluronic acid-based matrices modulates growth factor retention kinetics and stem cell fate. J Controlled Rel. 2015;209:308-16.

[145] Chen S, Han Y, Sun C, Dai L, Yang S, Wei Y, et al. Effect of molecular weight of hyaluronan on zein-based nanoparticles: fabrication, structural characterization and delivery of curcumin. Carbohydr Polym. 2018;201:599-607.

[146] Martínez-Sanz E, Ossipov DA, Hilborn J, Larsson S, Jonsson KB, Varghese OP. Bone reservoir: injectable hyaluronic acid hydrogel for minimal invasive bone augmentation. J Controlled Rel. 2011;152(2):232-40.

[147] Lee H-J, Heo Y, Park K-T, Kim E-H, Ito Y, Song K-S, et al. The immobilization of bone morphogenetic protein-2 via photo curable azidophenyl hyaluronic acid on a titanium surface and providing effect for cell differentiation. Macromol Res. 2014;22(2):173-8.

[148] Choi YH, Kim SH, Kim IG, Lee JH, Kwon SK. Injectable basic fibroblast growth factor-loaded alginate/hyaluronic acid hydrogel for rejuvenation of geriatric larynx. Acta Biomater. 2019;89:104-14.

[149] Nath SD, Abueva C, Kim B, Lee BT. Chitosan-hyaluronic acid polyelectrolyte complex scaffold crosslinked with genipin for 
immobilization and controlled release of BMP-2. Carbohydr Polym. 2015;115:160-9.

[150] Chung EJ, Chien KB, Aguado BA, Shah RN. Osteogenic potential of BMP-2-releasing self-assembled membranes. Tissue Eng Part A. 2013;19(23-24):2664-73.

[151] Lee J, Yoo KC, Ko J, Yoo B, Shin J, Lee S-J, et al. Hollow hyaluronic acid particles by competition between adhesive and cohesive properties of catechol for anticancer drug carrier. Carbohydr Polym. 2017;164:309-16.

[152] Bae MS, Yang DH, Lee JB, Heo DN, Kwon Y-D, Youn IC, et al. Photo-cured hyaluronic acid-based hydrogels containing simvastatin as a bone tissue regeneration scaffold. Biomaterials. 2011;32(32):8161-71.

[153] Schmidt JR, Vogel S, Moeller S, Kalkhof S, Schubert K, von Bergen $M$, et al. Sulfated hyaluronic acid and dexamethasone possess a synergistic potential in the differentiation of osteoblasts from human bone marrow stromal cells. J Cell Biochem. 2019;120(5):8706-22.

[154] Abourehab MAS. Hyaluronic acid modified risedronate and teriparatide co-loaded nanocarriers for improved osteogenic differentiation of osteoblasts for the treatment of osteoporosis. Curr Pharm Des. 2019;25(27):2975-88.

[155] Kettenberger U, Luginbuehl V, Procter P, Pioletti DP. In vitro and in vivo investigation of bisphosphonate-loaded hydroxyapatite particles for peri-implant bone augmentation. J Tissue Eng Regenerative Med. 2017;11(7):1974-85.

[156] Zhang K, Lin S, Feng Q, Dong C, Yang Y, Li G, et al. Nanocomposite hydrogels stabilized by self-assembled multivalent bisphosphonate-magnesium nanoparticles mediate sustained release of magnesium ion and promote in situ bone regeneration. Acta Biomater. 2017;64:389-400.

[157] Chang Y-L, Lo Y-J, Feng S-W, Huang Y-C, Tsai H-Y, Lin C-T, et al. Bone healing improvements using hyaluronic acid and hydroxyapatite/beta-tricalcium phosphate in combination: an animal study. BioMed Res Int. 2016;2016:8301624.
[158] Chen X, Meng Y, Wang Y, Du C, Yang C. A biomimetic material with a high bio-responsibility for bone reconstruction and tissue engineering. J Biomater Sci, Polym Ed. 2011;22(1-3):153-63.

[159] Souza JCM, Sordi MB, Kanazawa M, Ravindran S, Henriques B, Silva FS, et al. Nano-scale modification of titanium implant surfaces to enhance osseointegration. Acta Biomater. 2019;94:112-31.

[160] Séon-Lutz M, Couffin A-C, Vignoud S, Schlatter G, Hébraud A. Electrospinning in water and in situ crosslinking of hyaluronic acid/cyclodextrin nanofibers: towards wound dressing with controlled drug release. Carbohydr Polym. 2019;207:276-87.

[161] Fischer RL, McCoy MG, Grant SA. Electrospinning collagen and hyaluronic acid nanofiber meshes. J Mater Sci: Mater Med. 2012;23(7):1645-54.

[162] Wu G, Feng C, Quan J, Wang Z, Wei W, Zang S, et al. In situ controlled release of stromal cell-derived factor- $1 \alpha$ and antimiR-138 for on-demand cranial bone regeneration. Carbohydr Polym. 2018;182:215-24.

[163] Wang Z, Wu G, Wei M, Liu Q, Zhou J, Qin T, et al. Improving the osteogenesis of human bone marrow mesenchymal stem cell sheets by microRNA-21-loaded chitosan/hyaluronic acid nanoparticles via reverse transfection. Int J Nanomed. 2016;11:2091-105.

[164] Wu G, Feng C, Hui G, Wang Z, Tan J, Luo L, et al. Improving the osteogenesis of rat mesenchymal stem cells by chitosanbased-microRNA nanoparticles. Carbohydr Polym. 2016;138:49-58.

[165] Dong J, Tao L, Abourehab MAS, Hussain Z. Design and development of novel hyaluronate-modified nanoparticles for combo-delivery of curcumin and alendronate: fabrication, characterization, and cellular and molecular evidences of enhanced bone regeneration. Int J Biol Macromolecules. 2018;116:1268-81. 